\title{
The GAPS programme with HARPS-N at TNG
}

\section{A comprehensive analysis of the XO-2 stellar and planetary systems ${ }^{\star}, \star \star$}

\author{
M. Damasso ${ }^{1,2}$, K. Biazzo ${ }^{3}$, A. S. Bonomo ${ }^{1}$, S. Desidera ${ }^{4}$, A. F. Lanza ${ }^{3}$, V. Nascimbeni ${ }^{4,5}$, M. Esposito ${ }^{6,7}$, \\ G. Scandariato ${ }^{3}$, A. Sozzetti ${ }^{1}$, R. Cosentino ${ }^{3,8}$, R. Gratton ${ }^{4}$, L. Malavolta ${ }^{5,9}$, M. Rainer ${ }^{10}$, D. Gandolfi ${ }^{3,11}$, E. Poretti ${ }^{10}$, \\ R. Zanmar Sanchez ${ }^{3}$, I. Ribas ${ }^{12}$, N. Santos ${ }^{13,14,15}$, L. Affer ${ }^{16}$, G. Andreuzzi ${ }^{8}$, M. Barbieri ${ }^{4}$, L. R. Bedin ${ }^{4}$, S. Benatti ${ }^{4}$, \\ A. Bernagozzi ${ }^{2}$, E. Bertolini ${ }^{2}$, M. Bonavita ${ }^{4}$, F. Borsa $^{10}$, L. Borsato $^{5}$, W. Boschin ${ }^{8}$, P. Calcidese ${ }^{2}$, A. Carbognani ${ }^{2}$, \\ D. Cenadelli ${ }^{2}$, J. M. Christille ${ }^{2,17}$, R. U. Claudi ${ }^{4}$, E. Covino ${ }^{18}$, A. Cunial ${ }^{5}$, P. Giacobbe ${ }^{1}$, V. Granata ${ }^{5}$, A. Harutyunyan ${ }^{8}$, \\ M. G. Lattanzi ${ }^{1}$, G. Leto ${ }^{3}$, M. Libralato ${ }^{4,5}$, G. Lodato ${ }^{19}$, V. Lorenzi ${ }^{8}$, L. Mancini $^{20}$, A. F. Martinez Fiorenzano ${ }^{8}$, \\ F. Marzari ${ }^{4,5}$, S. Masiero ${ }^{4,5}$, G. Micela ${ }^{16}$, E. Molinari ${ }^{8,21}$, M. Molinaro ${ }^{22}$, U. Munari ${ }^{4}$, S. Murabito ${ }^{6,7}$, I. Pagano ${ }^{3}$, \\ M. Pedani ${ }^{8}$, G. Piotto ${ }^{4,5}$, A. Rosenberg ${ }^{6,7}$, R. Silvotti ${ }^{1}$, and J. Southworth ${ }^{23}$ \\ (Affiliations can be found after the references)
}

Received 13 November 2014 / Accepted 23 December 2014

\begin{abstract}
Aims. XO-2 is the first confirmed wide stellar binary system where the almost twin components $\mathrm{XO}-2 \mathrm{~N}$ and $\mathrm{XO}-2 \mathrm{~S}$ have planets, and it is a peculiar laboratory in which to investigate the diversity of planetary systems. This stimulated a detailed characterization study of the stellar and planetary components based on new observations.

Methods. We collected high-resolution spectra with the HARPS-N spectrograph and multi-band light curves. Spectral analysis led to an accurate determination of the stellar atmospheric parameters and characterization of the stellar activity, and high-precision radial velocities of XO-2N were measured. We collected 14 transit light curves of $\mathrm{XO}-2 \mathrm{Nb}$ used to improve the transit parameters. Photometry provided accurate magnitude differences between the stars and a measure of their rotation periods.

Results. The iron abundance of XO- $2 \mathrm{~N}$ was found to be +0.054 dex greater, within more than $3 \sigma$, than that of XO- $2 \mathrm{~S}$. The existence of a longterm variation in the radial velocities of XO- $2 \mathrm{~N}$ is confirmed, and we detected a turnover with respect to previous measurements. We suggest the presence of a second massive companion in an outer orbit or the stellar activity cycle as possible causes of the observed acceleration. The latter explanation seems more plausible with the present dataset. We obtained an accurate value of the projected spin-orbit angle for the XO-2N system $\left(\lambda=7^{\circ} \pm 11^{\circ}\right)$, and estimated the real 3D spin-orbit angle $\left(\psi=27_{-27}^{+12}\right.$ degrees $)$. We measured the XO-2 rotation periods, and found a value of $P=41.6 \pm 1.1$ days in the case of $\mathrm{XO}-2 \mathrm{~N}$, in excellent agreement with the predictions. The period of XO-2S appears shorter, with an ambiguity between 26 and 34.5 days that we cannot solve with the present dataset alone. The analysis of the stellar activity shows that XO-2N appears to be more active than the companion, perhaps because we sampled different phases of their activity cycle, or because of an interaction between XO-2N and its hot Jupiter that we could not confirm.
\end{abstract}

Key words. stars: individual: XO-2 - stars: fundamental parameters - stars: abundances - planetary systems - techniques: radial velocities techniques: photometric

\section{Introduction}

The diversity of the more than 1700 extrasolar planets discovered so $\operatorname{far}^{1}(\sim 850$ of which are in multi-planet systems; Rowe et al. 2014), from their orbital architectures to the astrophysical

\footnotetext{
* Based on observations made (i) with the Italian Telescopio Nazionale Galileo (TNG), operated on the island of La Palma by the INAF - Fundacion Galileo Galilei (Spanish Observatory of Roque de los Muchachos of the IAC); (ii) with the Copernico and Schmidt telescopes (INAF - Osservatorio Astrofisico di Padova, Asiago, Italy); (iii) with the IAC-80 telescope at the Teide Observatory (Instituto de Astrofísica de Canarias, IAC); (iv) at the Serra la Nave "M.G. Fracastoro" Astronomical Observatory (INAF - Osservatorio Astrofisico di Catania); (v) at the Astronomical Observatory of the Autonomous Region of the Aosta Valley (OAVdA).

$\star \star$ RV data (including Table 7) and photometry data are only available at the CDS via anonymous ftp to cdsarc.u-strasbg. fr $(130.79 .128 .5)$ or via

http://cdsarc.u-strasbg.fr/viz-bin/qcat?]/A+A/575/A111

1 NASA exoplanet archive, http://exoplanetarchive.ipac. caltech.edu/
}

environments where they reside, is a very complex issue that needs to be investigated in order to understand the mechanisms of their formation and dynamical evolution.

In the era of comparative exo-planetology, a fundamental approach for the understanding of the different exoplanetary properties relies on the characterization of planet-host stars and statistical analysis of the planetary system frequency. Several studies have shown that the properties of the planetary system architectures and physical characteristics of the planets depend on stellar properties, such as the mass (Johnson et al. 2010; Bonfils et al. 2013) and the metallicity (Sozzetti et al. 2009; Santos et al. 2011; Mortier et al. 2012). Moreover, in recent years the search for exoplanets has been extended in environments such as giant stars (e.g. Kepler-91, Lillo-Box et al. 2014b,a), and open and globular clusters, and planetary companions have been found (see e.g. Quinn et al. 2012; Meibom et al. 2013, and references therein).

Binary systems are interesting astrophysical environments in which to search for planets. Considering that nearly half of the solar-type stars are gravitationally bound with at least another 
stellar companion (e.g. Raghavan et al. 2010; Duchêne \& Kraus 2013), binary systems are naturally a typical environment to be explored for studying the processes of planet formation and evolution leading to very different planetary architectures.

Planet formation in a binaries has been considered by various authors. For coplanar orbits between the disk and the binary, the general expectation (e.g. Marzari \& Scholl 2000; Kley $\&$ Nelson 2008; Marzari et al. 2012) is that the tidal effect of the companion would induce an eccentricity growth in the planetesimal population that might inhibit planet formation if the binary separation is $\lesssim 50$ AU. In this configuration even the formation of icy planetesimals is jeopardized by the high temperatures in the disks and the formation of breaking waves (Nelson 2000; Picogna \& Marzari 2013). The situation is more complicated for misaligned systems where the planetesimals and gas disks precess around the binary orbital plane. Planetesimals naturally develop strong differential precession that would inhibit planet formation by increasing the velocity dispersion (Marzari et al. 2009). On the other hand, if the gas disk is radially narrow (which is expected for separation of the order of $50 \mathrm{AU}$ because of tidal truncation), it will precess rigidly and would drag the planetesimals into rigid precession, mitigating the previous effect (Fragner et al. 2011), unless the inclination is too large for Lidov-Kozai effects to become important. In this case the effects should also be relevant mostly for binary separations smaller than 50-100 AU. For wider, misaligned binaries the gas disk is expected instead to reach a quasi-steady, non-precessing warped configuration (see Facchini et al. 2013 for the case of a circumbinary disk). If the warp is located in the planet formation region, it might affect the process, although such effects have not yet been studied.

Several examples of planets orbiting only one of the binary components, defined as $S$-type planets, are known (e.g. Roell et al. 2012; Mugrauer et al. 2014; and Thebault \& Haghighipour 2014 , for a recent review). The existence of $S$-type planets raises several questions: how can the presence of a stellar companion affect the formation, survival, and dynamical evolution of such planets? Is it possible to identify which properties of a stellar system most influence the physical characteristics of the hosted planets? Does any constraint exist with respect to the case of isolated stars, or any dependence on the physical separation of the binary components? Observational difficulties exist in searching for S-type planets in multiple stellar systems (Eggenberger \& Udry 2010), in particular those with a very small angular separation (less then $\sim 2^{\prime \prime}$ ), since, for instance, the components cannot be observed as two isolated targets in the spectrograph's fibre or slit. In many cases of confirmed extrasolar planets the presence of a stellar companion to the host star was discovered after the detection of the planet (e.g. Roell et al. 2012). Binarity poses a major challenge in understanding how such planets could exist and their evolutionary history in the stellar system.

Very wide binaries, with semi-major axes of the order of $10^{3} \mathrm{AU}$ and sky projected angular separations of several arcsec, offer the best opportunity and fewer observational complications to search for planets orbiting each component. At the same time they allow an accurate determination of the properties of the individual stars. Despite the large separation between the stellar components, these systems are an interesting subject for dynamical studies also related to the presence of planets.

Kaib et al. (2013) and Kaib \& Raymond (2014) studied the perturbations produced by passing stars and the tidal effects of the Milky Way on the orbits of very wide binary stars. Interestingly, they found that occasionally the orbits could become extremely eccentric, resulting in the collision of the binary components or, less dramatically, they could evolve toward close or contact systems. Moreover, simulations by Kaib et al. (2013) indicate that very wide binary companions may often strongly reshape planetary systems after their formation by causing the ejection of planets from the system or heavily changing the orbital eccentricities of those that survive. Another intriguing example of a plausible fate for planets in wide binaries is the "bouncing" scenario investigated by Moeckel \& Veras (2012). They found that in 45 to 75 per cent of the cases a planet, initially orbiting one of the binary components and then ejected from its native system by planet-planet scattering, could jump up and down passing from the gravitational influence of one star to the other for more than a Myr. This situation may trigger orbital instability among existing planets around the companion, and in some cases result in an exchange of planets between the two stars, when both host multiple planetary systems.

At present, few very wide binaries are known where a planetary system was discovered around one of the stellar components. The star HD 20782 is the binary companion of HD 20781, with a projected separation of $\sim 9000 \mathrm{AU}$, and it hosts a Jupiter-mass planet on a very eccentric orbit at $\sim 1.4 \mathrm{AU}$ $(\text { Jones et al. 2006) })^{2}$. The very high eccentricity reported in the literature $(e=0.97$ ) could be the result of significant perturbations experienced by the planet during the evolution of the system and might be due to the stellar companion. In this binary system, where both the components can be well analysed separately, a comparative study of the physical properties of the pair could help explain the nature of the planetary system. To investigate any existing link between the planet formation mechanisms and the chemical composition of the host stars, Mack et al. (2014) performed a detailed elemental abundance analysis of the atmospheres of HD 20781/82, by considering that both stars host planets. This kind of study, when at least one component hosts a close-in giant planet, could result in the evidence of chemical imprints left in the parent star suggesting the ingestion of material from the circumstellar disk (and possibly also from planets) driven by the dynamical evolution of the planet orbit. When a star with a close-in giant planet is found to be enriched with elements of high condensation temperature, as suggested by Schuler et al. (2011b), this can be related to the inward migration of the planet from the outer regions of the circumstellar disk, where it formed, to the present position closer to the star (Ida \& Lin 2008; Raymond et al. 2011). Mack et al. (2014) found quite significant positive trends for both stars with the condensation temperature among the elemental abundances, and suggested that the host stars accreted rocky bodies with mass between 10 and $20 M_{\oplus}$ initially formed inside the location of the detected planets. Searches for abundance anomalies possibly caused by the ingestion of planetary material by the central star were also conducted by (Desidera et al. 2004, 2007; and references therein) for a sample of wide binaries. These authors found that the amount of iron accreted by the companion that is nominally richer in metal (in binaries with components having $T_{\text {eff }}>5500 \mathrm{~K}$ ) is comparable to the estimates of the rocky material accreted by the Sun during its main-sequence lifetime, and therefore concluded that the metal enrichment due to the ingestion of material of planetary origin is not a common event.

A representative case of a very wide binary with one component hosting a close-in giant planet is that of the XO-2 system (projected separation $\sim 31^{\prime \prime}$ ), where the star $\mathrm{XO}-2 \mathrm{~N}$ is orbited by

2 HD 20781 should host two Neptune-mass planets within $0.3 \mathrm{AU}$, as reported by Mayor et al. (2011), but the discovery has not yet been confirmed and published. 
the transiting hot Jupiter XO-2Nb (Burke et al. 2007). An elemental abundance analysis of XO-2N and its companion XO-2S was performed by Teske et al. (2013), who determined the stellar abundances of carbon and oxygen, as well as iron and nickel. Their goal was to probe the potential effects that planet formation and evolution might have had on the chemical composition of $\mathrm{XO}-2 \mathrm{~N}$, where the companion $\mathrm{XO}-2 \mathrm{~S}$ was treated as a nonhosting planet star. Later, Desidera et al. (2014) discovered that the star XO-2S actually hosts a planetary system formed by a planet slightly more massive than Jupiter orbiting at $0.48 \mathrm{AU}$ and a Saturn-mass planet at $0.13 \mathrm{AU}$ (with cautious evidence of a long-term trend in the radial velocities time series possibly due to an outer companion, yet of unknown nature). The discovery is of particular relevance because it represents the first confirmed case of a very wide binary in which both components host planets (see Fig. 1 for a sketch of the XO-2 planetary systems). The XO-2 binary offers a unique opportunity to explore the diversity of planet formation mechanisms by considering that, although the parent stars are almost equal in their main physical properties, the planetary systems are different. This finding has consequently motivated a detailed comparative study of the whole system, which is the subject of this paper, through the use of new high-resolution and high signal-to-noise (S/N) HARPS-N spectra and dedicated differential photometry. It is crucial to highlight any existing difference between the properties of the two stars that could have played a distinctive role in the formation processes of the planetary systems: any observed difference, if correctly interpreted, can tell a part of that story. While the analysis of the XO-2 system was still in progress, the discovery of two hot Jupiters was announced, one around each of the twin stars of the WASP-94 wide binary (Neveu-VanMalle et al. 2014). They are metal rich $([\mathrm{Fe} / \mathrm{H}] \sim 0.25 \mathrm{dex})$, F-type components with a projected separation of $\sim 2700$ AU. This system, with host star properties and planetary system architectures different from those of XO-2, indeed makes it more complicated to understand the role of stellar multiplicity on the planet formation and evolution.

This paper is organized as follows. We first describe in Sect. 2 the spectroscopic and photometric datasets used in this study. We present in Sect. 3 a new determination of the basic stellar parameters based on HARPS-N spectra, as well as results of a detailed and homogeneous analysis aimed at searching for differences in the iron abundance between the XO-2 components with high level of confidence. In Sect. 4 we present and discuss results from a photometric follow-up of the two stars, including the measurement of the stellar rotation periods and the modelling of a new dataset of 14 transits of the planet XO-2Nb. Section 5 is dedicated to the $\mathrm{XO}-2 \mathrm{~N}$ planetary system; we present a new analysis of the radial velocity time series, thanks to which we confirm the existence of an acceleration with a not yet established cause. In the same section we also present new observation and analysis of the Rossiter-McLaughlin effect, better constraining the value of the projected spin-orbit angle $\lambda$. A detailed analysis of the stellar activity for the $\mathrm{XO}-2$ components is presented and discussed in Sect. 6. We conclude with Sect. 7, where we speculate about the evolution and long-term stability of the $\mathrm{XO}-2$ planetary systems.

\section{Observations and data reduction methods}

\subsection{Spectroscopy}

The spectra of the XO-2 components analysed in this work were collected with the high-resolution HARPS-N spectrograph
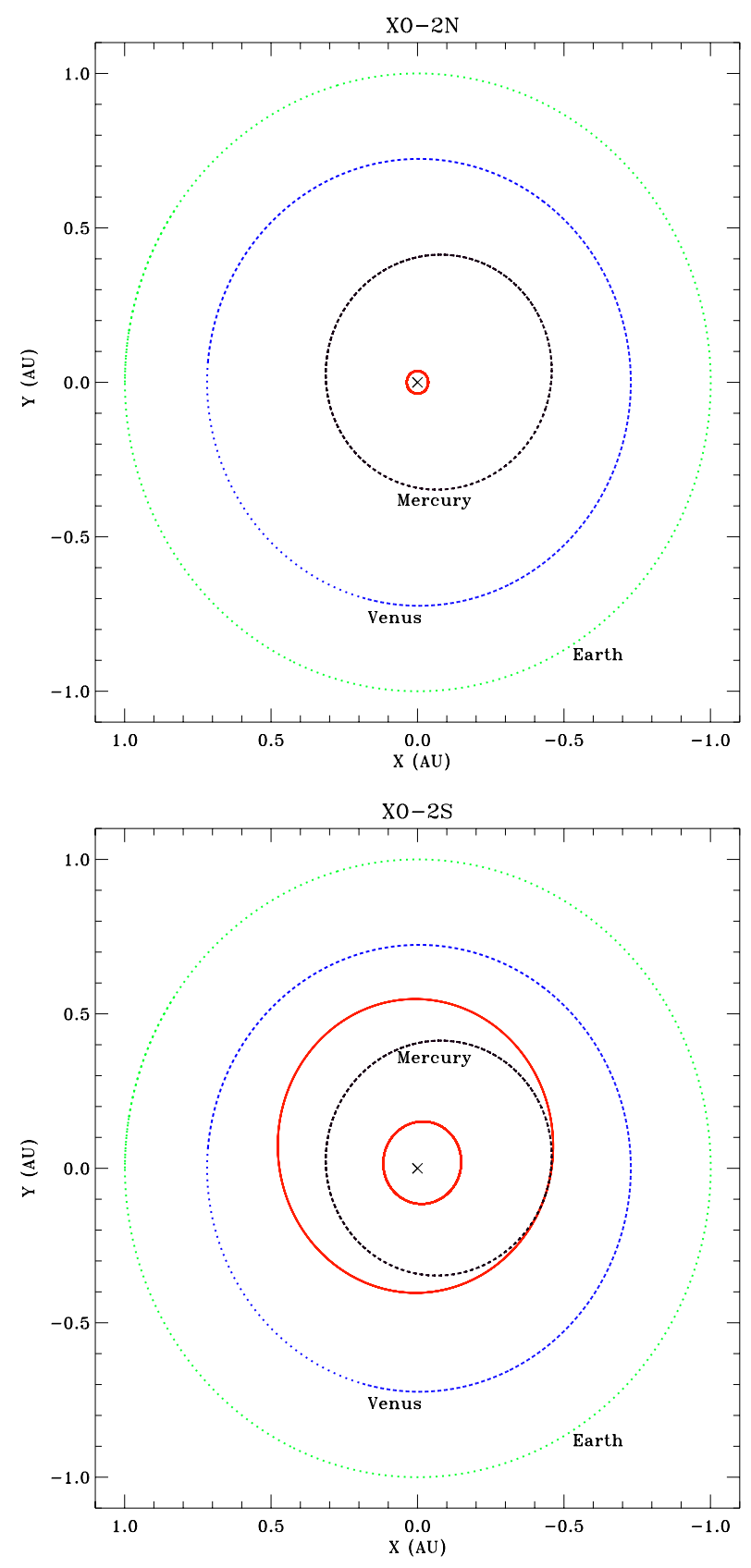

Fig. 1. Comparison between the planetary systems orbiting the XO-2 binary stars and the architecture of the inner Solar System. The case of $\mathrm{XO}-2 \mathrm{~N}$ is shown in the upper panel, while that of $\mathrm{XO}-2 \mathrm{~S}$ is represented in the lower one. In both plots, the red ellipses (solid lines) represent the orbits of the XO-2 planets. The orbits of Mercury, Venus and the Earth are indicated with black, blue and green dotted lines respectively, also labelled with the planet names. The cross symbol in the middle of each plot indicates the star location.

(Cosentino et al. 2012) installed at the Telescopio Nazionale Galileo (TNG) on La Palma (Canary islands). The observations were carried out in the framework of the large programme Global Architecture of Planetary Systems (GAPS; Covino et al. 2013; Desidera et al. 2013).

For the star XO-2N we collected 43 spectra between November 20, 2012, and October 4, 2014, while the companion XO-2S was observed at 63 individual epochs between April 21, 2013, and May 10, 2014. The Th-Ar simultaneous calibration was not used in order to avoid contamination by the lamp lines. 
This has no significant impact on the measurements of the radial velocity, because the drift correction with respect to the reference calibration shows a dispersion of $0.8 \mathrm{~m} \mathrm{~s}^{-1}$, which is smaller than the median radial velocity (RV) uncertainty due to photon-noise, i.e. $2.0 \mathrm{~m} \mathrm{~s}^{-1}$ for XO-2N and $2.2 \mathrm{~m} \mathrm{~s}^{-1}$ for XO-2S. The reduction of the spectra and the RV measurements were obtained for both components using the latest version (Nov. 2013) of the HARPS-N instrument Data Reduction Software pipeline and applying a K5 mask. The measurement of the RVs is based on the weighted cross-correlation function (CCF) method (Baranne et al. 1996; Pepe et al. 2002).

\subsection{Photometry}

We collected and analysed new photometric light curves for both components of the XO-2 system with three different facilities. In particular, XO-2S was intensively monitored with the HARPS-N spectrograph. When it became clear that the star had planetary companions, we started a dedicated photometric follow-up of the target to estimate the stellar rotational period, further characterizing its level of activity, and to look for possible transits of its exoplanets.

\subsubsection{The APACHE dataset}

A follow-up of the XO-2 field was conducted for 42 nights between December 2, 2013, and April 8, 2014, with one of the $40 \mathrm{~cm}$ telescopes composing the APACHE array (Sozzetti et al. 2013), based at the Astronomical Observatory of the Autonomous Region of the Aosta Valley (OAVdA, +45.7895 $\mathrm{N},+7.478$ E, 1650 m.a.s.l.). Each telescope is a Carbon Truss f/8.4 Ritchey-Chrétien equipped with a GM2000 10-MICRON mount and a FLI Proline PL1001E-2 CCD Camera, with a pixel scale of $1.5^{\prime \prime} / \mathrm{pixel}$ and a field of view of $26^{\prime} \times 26^{\prime}$. The observations were carried out using a Johnson-Cousins $I$ filter. The images were reduced with the standard pipeline TEEPEE written in IDL $^{3}$ and regularly used for the APACHE data (see Giacobbe et al. 2012). The APACHE data were used to tentatively derive the rotation period of the two stars and to monitor their magnitude difference in $I$ band, as described in Sect. 4.

\subsubsection{The TASTE dataset}

Since February 2011, several transits of the hot Jupiter XO-2Nb have been observed at high temporal cadence with the $182 \mathrm{~cm}$ and the Schmidt 92/67 telescopes at the Asiago Astrophysical Observatory $(+45.8433 \mathrm{~N},+11.5708 \mathrm{E}, 1366$ m.a.s.l.) in the framework of the TASTE Project (The Asiago Search for Transit time variations of Exoplanets; Nascimbeni et al. 2011). A subset of these measurements was used to estimate the magnitude differences of the two XO-2 binary components in the $R_{\mathrm{c}}$ band. Two additional transits were collected with the IAC- 80 telescope at the Teide Observatory, using the CAMELOT CCD imager (see Nascimbeni et al. 2013, for a description of the set-up), and one supplementary light curve was obtained with the same telescope but using the TCP camera (Tromsö CCD Photometer; Østensen \& Solheim 2000). All these observations, for a total of 14 light curves, were carried out in the Cousin/Bessell $R$ band and were analysed together to improve the determination of the parameters of $\mathrm{XO}-2 \mathrm{Nb}$.

\footnotetext{
3 Registered trademark of Exelis Visual Information Solutions.
}

\subsubsection{The Serra la Nave dataset}

The XO-2 system was also observed for five nights, between May 6, 2013, and May 7, 2014, with the robotic $80 \mathrm{~cm}$ f/8 Ritchey-Chrétien telescope APT2, operated by the INAFCatania Astrophysical Observatory and located at Serra la Nave (SLN, +37.692 N, +14.973 E, 1725 m.a.s.1.). The telescope is equipped with a CCD detector Apogee Alta U9000 (3 K $\times$ $3 \mathrm{~K}$ pixels) operated in binning $2 \times 2$, corresponding to a pixel scale $0.76 \mathrm{arcsec} / \mathrm{pixel}$. The data were reduced with the IRAF package $^{4}$ using the standard procedure for overscan, bias and dark subtraction, and flat fielding. The aperture photometry was done with the software SExtractor (Bertin \& Arnouts 1996). The data of SLN were collected in the BVRI bands and used to measure the magnitude differences between the two stars.

\section{Stellar parameters}

\subsection{Spectroscopic analysis of the individual stars}

The components of the XO-2 binary system have a separation of $\sim 31$ arcsec (corresponding to a projected distance of $\sim 4600 \mathrm{AU}$ assuming a distance of $\sim 150 \mathrm{pc}$, as estimated by Burke et al. 2007), and share, within the uncertainties, the proper-motion vector as listed in the UCAC4 catalogue. Essential information about the $\mathrm{XO}-2$ system is presented in Table 1. A standard analysis of the HARPS-N spectra was performed with different methods to derive the stellar atmospheric parameters of the two components, with those for XO-2S first presented in Desidera et al. (2014). Table 2 summarizes our results, which represent the weighted averages of the individual measurements. We used implementations of both the equivalent width and the spectral synthesis methods, as described in Biazzo et al. (2012), Santos et al. (2013), and Esposito et al. (2014). It is interesting to note that the spectroscopically derived effective temperature difference between the two stars is in good agreement with the result obtained from photometric measurements. In fact, following the same analysis performed by Munari et al. (2014) for half a million stars of the RAVE spectroscopic survey, if we assume a colour excess of $E(B-V)=0.02 \mathrm{mag}$ (as explained in Sect. 3.3) the XO-2S star is $60 \pm 33 \mathrm{~K}$ hotter than the companion. The stellar mass, radius, and age were determined by comparing our measured effective temperature, iron abundance, and surface gravity with the Yonsei-Yale (Y-Y) evolutionary tracks (Demarque et al. 2004) through the $\chi$-square statistics (Santerne et al. 2011). The adopted errors include an extra $5 \%$ in mass and $3 \%$ in radius added in quadrature to the formal errors to take systematic uncertainties in stellar models into account (Southworth 2011). Taking advantage of the fact that the planet $\mathrm{XO}-2 \mathrm{Nb}$ transits its parent star, we derived a second, slightly more accurate estimate for the mass, radius, and age of XO-2N, using the stellar density information as determined from the analysis of the transit light curves (e.g. Sozzetti et al. 2007).

\subsection{Differential spectral analysis}

Thanks to the high-quality of the HARPS-N spectra, we performed a detailed and homogeneous spectral analysis of $\mathrm{XO}-2 \mathrm{~N}$ and $\mathrm{XO}-2 \mathrm{~S}$ to search for possible differences in the iron abundance between the components. The average values for $[\mathrm{Fe} / \mathrm{H}]$ obtained from the analysis of the individual stars (Table 2) indicate that the northern component is characterized

\footnotetext{
4 http://iraf.noao.edu/
} 
M. Damasso et al.: The GAPS programme with HARPS-N at TNG. V.

Table 1. Basic information about the XO-2 stellar binary system.

\begin{tabular}{|c|c|c|c|}
\hline Parameter & $\mathrm{XO}-2 \mathrm{~N}$ & $\mathrm{XO}-2 \mathrm{~S}$ & Ref. \\
\hline UCAC4 ID & $702-047113$ & $702-047114$ & (1) \\
\hline RA (J2000) & 07:48:06.471 & 07:48:07.479 & (1) \\
\hline Dec (J2000) & $+50: 13: 32.91$ & $+50: 13: 03.25$ & (1) \\
\hline Near UV (mag) & $18.153 \pm 0.047$ & $17.988 \pm 0.044$ & (2) \\
\hline$B$ (mag) & $12.002 \pm 0.015$ & $11.927 \pm 0.015$ & (3) \\
\hline$V(\mathrm{mag})$ & $11.138 \pm 0.026$ & $11.086 \pm 0.025$ & (3) \\
\hline$R_{\mathrm{c}}(\mathrm{mag})$ & $10.669 \pm 0.020$ & $10.631 \pm 0.019$ & (3) \\
\hline$I_{\mathrm{c}}(\mathrm{mag})$ & $10.243 \pm 0.012$ & $10.216 \pm 0.013$ & (3) \\
\hline$J$ (mag) & $9.744 \pm 0.022$ & $9.742 \pm 0.022$ & (4) \\
\hline$H(\mathrm{mag})$ & $9.340 \pm 0.026$ & $9.371 \pm 0.027$ & (4) \\
\hline$K_{\mathrm{s}}(\mathrm{mag})$ & $9.308 \pm 0.021$ & $9.272 \pm 0.021$ & (4) \\
\hline Distance (pc) & $145.5_{-3.0}^{+2.9}$ & $148.0_{-2.9}^{+2.8}$ & (5) \\
\hline$\mu_{\alpha}\left(\operatorname{mas} \mathrm{yr}^{-1}\right)$ & $-31.9 \pm 3.0$ & $-29.9 \pm 3.6$ & (1) \\
\hline$\mu_{\delta}\left({\left.\operatorname{mas~} \mathrm{yr}^{-1}\right)}^{-1}\right.$ & $-156.2 \pm 3.3$ & $-156.2 \pm 3.6$ & (1) \\
\hline$U\left(\mathrm{~km} \mathrm{~s}^{-1}\right)$ & $70.39_{-0.81}^{+0.79}$ & $71.05_{-0.76}^{+0.75}$ & (5) \\
\hline$V\left(\mathrm{~km} \mathrm{~s}^{-1}\right)$ & $-76.0_{-2.0}^{+2.0}$ & $-77.65_{-1.88}^{+1.92}$ & (5) \\
\hline$W\left(\mathrm{~km} \mathrm{~s}^{-1}\right)$ & $-2.91_{-0.66}^{+0.67}$ & $-3.46_{-0.62}^{+0.64}$ & (5) \\
\hline \multicolumn{4}{|c|}{ Relative magnitudes (XO-2N $-\mathrm{XO}-2 \mathrm{~S})$} \\
\hline$\Delta B(\mathrm{mag})$ & \multicolumn{2}{|c|}{$0.075 \pm 0.002$} & (6) \\
\hline$\Delta V(\mathrm{mag})$ & \multicolumn{2}{|c|}{$0.057 \pm 0.002$} & (6) \\
\hline \multirow[t]{2}{*}{$\Delta R_{\mathrm{c}}(\mathrm{mag})$} & \multicolumn{2}{|c|}{$0.040 \pm 0.002$} & $(6)$ \\
\hline & \multicolumn{2}{|c|}{$0.041 \pm 0.004$} & (7) \\
\hline$\Delta r^{\prime}(\mathrm{mag})$ & \multicolumn{2}{|c|}{$0.038 \pm 0.001$} & (8) \\
\hline$\Delta I_{\mathrm{c}}(\mathrm{mag})$ & \multicolumn{2}{|c|}{$0.031 \pm 0.003$} & (6) \\
\hline & \multicolumn{2}{|c|}{$0.030 \pm 0.006$} & $(9)$ \\
\hline
\end{tabular}

References. (1) UCAC4 catalogue (Zacharias et al. 2013); (2) Bianchi et al. (2011); (3) APASS all-sky survey (Henden \& Munari 2014); (4) 2MASS catalogue (Skrutskie et al. 2006); (5) this work; (6) this work (Serra La Nave Observatory); (7) this work (TASTE project); (8) Kundurthy et al. (2013), $\lambda_{0}=626 \mathrm{~nm}, 4$ epochs; (9) this work (APACHE project).

by higher iron abundance, but the two estimates are compatible within the uncertainties. In order to validate this difference with a high level of confidence, we determined the difference in $[\mathrm{Fe} / \mathrm{H}]$ very accurately with a dedicated analysis. Together with the spectra of both targets, we also acquired three solar spectra through observations of the asteroid Vesta. This allowed us to perform a differential analysis for each component with respect to the Sun, thus avoiding the contribution from the uncertainties in atomic parameters, such as the transition probabilities. All HARPS-N spectra were shifted in wavelength and then coadded in order to obtain very high $S / N$ spectra $(\sim 180-250$ at $\lambda \sim 6700 \AA$ ). We performed the spectral analysis following three main steps:

i. Analysis based on equivalent widths. We measured the equivalent widths (EWs) of iron lines on 1-D spectra using the splot task in IRAF, and took care to trace as closely
Table 2. Stellar parameters for the XO-2 components derived from the analysis of the HARPS-N spectra and from stellar evolutionary tracks.

\begin{tabular}{cccc}
\hline \hline Parameter & XO-2N & XO-2S & Note \\
\hline$T_{\text {eff }}[\mathrm{K}]$ & $5332 \pm 57$ & $5395 \pm 54$ & \\
$\log g[\mathrm{cgs}]$ & $4.44 \pm 0.08$ & $4.43 \pm 0.08$ & \\
{$[\mathrm{Fe} / \mathrm{H}][\mathrm{dex}]$} & $0.43 \pm 0.05$ & $0.39 \pm 0.05$ & \\
Microturb. $\xi\left[\mathrm{km} \mathrm{s}^{-1}\right]$ & $0.88 \pm 0.11$ & $0.90 \pm 0.10$ & \\
$V \sin I_{\star}\left[\mathrm{km} \mathrm{s}^{-1}\right]$ & $1.07 \pm 0.09$ & $1.5 \pm 0.3$ & \\
Mass $\left[M_{\odot}\right]$ & $0.97 \pm 0.05$ & $0.98 \pm 0.05$ & $(1)$ \\
& $0.96 \pm 0.05$ & - & $(2)$ \\
Radius $\left[R_{\odot}\right]$ & $1.01_{-0.07}^{+0.1}$ & $1.02_{-0.06}^{+0.09}$ & $(1)$ \\
& $0.998_{-0.032}^{+0.033}$ & - & $(2)$ \\
Age $[\mathrm{Gyr}]$ & $7.9_{-3.0}^{+2.3}$ & $7.1_{-2.9}^{+2.5}$ & $(1)$ \\
& $7.8_{-1.3}^{+1.2}$ & - & $(2)$ \\
Luminosity $\left[L_{\odot}\right]$ & $0.70 \pm 0.04$ & $0.79 \pm 0.14$ for XO-2N: (2) \\
& & & for XO-2S: (1) \\
\hline
\end{tabular}

Notes. The adopted values represent the weighted mean of individual measurements obtained with different methods (except for the projected velocity $V \sin I_{\star}$, for which we adopt the measurement derived from the Rossiter-McLaughlin effect), and their associated uncertainties are, for a more conservative estimate, the average of the uncertainties of the individual values. (1) Matching the $T_{\text {eff }},[\mathrm{Fe} / \mathrm{H}]$, and $\log g$ to the Yonsei-Yale evolutionary tracks. (2) Matching the $T_{\mathrm{eff}},[\mathrm{Fe} / \mathrm{H}]$, and stellar density to the Yonsei-Yale evolutionary tracks.

as possible the same position in the continuum level for the spectra of both components and the asteroid Vesta. We considered the line-list from Biazzo et al. (2012), and adopted the same procedure as these authors for the differential analysis relative to the Sun, the rejection criteria of bad lines, the line broadening mechanisms, the model atmospheres, the measurement of stellar parameters and iron abundances, and their corresponding uncertainties. We refer to that paper and to Biazzo et al. (2011) for detailed descriptions of the method. In brief, we used the 2013 version of the MOOG code (Sneden 1973) and considered the abfind driver, assuming local thermodynamic equilibrium (LTE). Initial stellar parameters were set to the solar values $\left(T_{\text {eff } \odot}=5770 \mathrm{~K}, \log g_{\odot}=4.44\right.$, and $\xi_{\odot}=$ $1.10 \mathrm{~km} \mathrm{~s}^{-1}$ ) for both components. Then, the final effective temperature $\left(T_{\text {eff }}\right)$ was determined by imposing the condition that the abundance from the Fe I lines $(\log n(\mathrm{Fe} \mathrm{I}))$ was independent of the line excitation potentials; the final microturbulence $(\xi)$ was determined by minimizing the slope of $\log n(\mathrm{Fe} \mathrm{I})$ vs. the reduced equivalent width $(E W / \lambda)$; and the final surface gravity $(\log g)$ was determined by imposing ionization equilibrium (i.e. $\log n(\mathrm{Fe} \mathrm{I})=\log n(\mathrm{Fe}$ II $)$ ). Plots of iron abundance $(\log n(\mathrm{Fe}))$ vs. excitation potential $(\chi)$ and reduced EW for both components are shown in Fig. 2, which shows that the correlations are close to zero, as required. Using the same procedure, we obtained $\log n(\mathrm{Fe} \mathrm{I})_{\odot}=\log n(\mathrm{Fe} \text { II })_{\odot}=7.53 \pm 0.05$ for the Sun. We point out that the exact values of the solar parameters are not crucial as we are performing a differential study for both components with respect to the Sun. Table 3 lists the final results of this procedure, where the errors in iron abundance include uncertainties in stellar parameters and in EW measurements. Results from this first homogeneous analysis seem to 
Table 3. Stellar atmospheric parameters and their differences for $\mathrm{XO}-2 \mathrm{~N}$ and $\mathrm{XO}-2 \mathrm{~S}$.

\begin{tabular}{cccc}
\hline \hline Parameter & XO-2N & XO-2S & $\Delta_{\mathrm{N}-\mathrm{S}}$ \\
\hline \multicolumn{4}{c}{ Analysis based on equivalent widths } \\
$T_{\text {eff }}[\mathrm{K}]$ & $5320 \pm 50$ & $5330 \pm 50$ & $-10 \pm 71$ \\
$\log g[\mathrm{cgs}]$ & $4.46 \pm 0.08$ & $4.41 \pm 0.12$ & $+0.05 \pm 0.14$ \\
$\xi\left[\mathrm{km} \mathrm{s}^{-1}\right]$ & $0.89 \pm 0.14$ & $0.93 \pm 0.04$ & $-0.04 \pm 0.15$ \\
{$[\mathrm{Fe} / \mathrm{H}]^{a}[\mathrm{dex}]$} & $0.37 \pm 0.07$ & $0.32 \pm 0.08$ & $+0.05 \pm 0.11$ \\
\multicolumn{4}{c}{ Line-by-line analysis } \\
$T_{\text {eff }}[\mathrm{K}]$ & $5290 \pm 18$ & $5322 \pm 25$ & $-32 \pm 31$ \\
$\log g[\mathrm{cgs}]$ & $4.43 \pm 0.10$ & $4.41 \pm 0.10$ & $+0.02 \pm 0.14$ \\
$\left.\xi[\mathrm{km} \mathrm{s})^{-1}\right]$ & $0.86 \pm 0.06$ & $0.93 \pm 0.05$ & $-0.07 \pm 0.08$ \\
{$[\mathrm{Fe} / \mathrm{H}]^{a}[\mathrm{dex}]$} & $0.37 \pm 0.07$ & $0.32 \pm 0.08$ & $+0.05 \pm 0.11$ \\
$T_{\text {eff }}(\mathrm{K}]$ & $5290 \pm 18$ & $5325 \pm 37$ & $-35 \pm 8$ \\
$\log g[\mathrm{cgs}]$ & $4.43 \pm 0.10$ & $4.420 \pm 0.094$ & $+0.010 \pm 0.020$ \\
$\xi\left[\mathrm{km} \mathrm{s}\right.$ s$\left.^{-1}\right]$ & $0.86 \pm 0.06$ & $0.93 \pm 0.03$ & $-0.07 \pm 0.07$ \\
{$[\mathrm{Fe} / \mathrm{H}]^{a}[\mathrm{dex}]$} & $0.37 \pm 0.07$ & $0.32 \pm 0.08$ & $+0.054 \pm 0.013$ \\
\hline
\end{tabular}

Notes. See discussion in Sect. 3.2. ${ }^{(a)}$ The iron abundance $[\mathrm{Fe} / \mathrm{H}]$ refers to the abundance of Fe I.

agree with those obtained with different methods of spectral analysis (Table 2).

ii. Line-by-line analysis. Since we are interested in a homogeneous analysis, we applied the same iterative method as in the first step, but here we considered a strict line-by-line analysis, i.e. the same lines were used for both components, without any implementation of line rejection criteria. This allowed us to avoid uncertainties related to both atomic parameters and measurements of equivalent widths, due to, e.g. blends and continuum level. In the end, the analysis was based on $75 \mathrm{Fe}$ I and $10 \mathrm{Fe}$ II lines. Thanks to such analysis, the errors in $T_{\text {eff }}$ were reduced substantially, as shown in Table 3.

iii. Differential analysis. Here, as stellar parameters of $\mathrm{XO}-2 \mathrm{~N}$, we considered the values derived in the line-by-line analysis, while $\mathrm{XO}-2 \mathrm{~S}$ was analysed differentially with respect to the companion, using the same line set and EWs as in the previous step. We applied the differential abundance method widely described in Gratton et al. (2001) and Desidera et al. (2004, 2006), which yields very accurate results when, in particular, the two components have very similar stellar parameters (as do the XO-2 components). In summary, the most important ingredient in this analysis is the difference in temperature between the components. In our case, we take advantage of the luminosity difference between the targets, as we can assume they are at the same distance from us. We considered as magnitude differences in different bands the mean values given in Sect. 4.1 and as bolometric corrections those derived by using the code provided by Casagrande \& VandenBerg (2014), which allows us to estimate the bolometric corrections in several photometric bands using $T_{\text {eff }},[\mathrm{Fe} / \mathrm{H}]$, and $\log g$ as primary inputs. Then, since the stars are on the main sequence, we were able to obtain accurate estimates of the difference $\Delta \log g$ between their surface gravities (see Eq. (6) in Desidera et al. 2006, where as stellar mass difference we assumed $0.01 M_{\odot}$, as given in Table 2). This allowed us to accurately derive their temperature difference ( $\left.\Delta T_{\text {eff }}\right)$ using the equilibrium of the iron ionization, because the difference between the abundances provided by the Fe I and Fe II lines is strongly sensitive to $T_{\text {eff }}$ (about $0.001 \mathrm{dex} / \mathrm{K}$; see Gratton et al. 2001). The final atmospheric parameters of the secondary were derived as differences with respect to those of the primary by means of an iterative procedure (because $\log g, \xi$, and $[\mathrm{Fe} / \mathrm{H}]$ depend on the assumed temperatures). We interrupted the procedure and considered the convergence reached when the grid stepsize was of $1 \mathrm{~K}$ in $T_{\text {eff }}, 0.001 \mathrm{dex}$ in $\log g$, and $0.01 \mathrm{~km} \mathrm{~s}^{-1}$ in $\xi$. No other $\sigma$ clipping was implemented here. The final adopted differences in stellar parameters are listed in Table 3, while Fig. 3 shows $\Delta[\mathrm{Fe} / \mathrm{H}]$ as a function of the excitation potential. The under-abundance in iron and the higher effective temperature of $\mathrm{XO}-2 \mathrm{~S}$ when compared to $\mathrm{XO}-2 \mathrm{~N}$ are confirmed by this procedure. All the differential parameters we obtained are affected by both internal and systematic errors. The internal error on $\Delta T_{\text {eff }}$ was derived from the lineby-line scatter of Fe I and Fe II and the errors in the other atmospheric parameters. The internal error in $\Delta \log g$ includes the uncertainties in the differences in mass, magnitude, bolometric correction, and effective temperature. The error in the microturbulence difference was obtained by summing in quadrature the error contributions in $\xi$ of each component. The error in the iron abundance difference was derived taking into account the uncertainties in all stellar parameters added quadratically. All errors in the differential analysis are very low, as summarized in Table 3 , and this demonstrates how efficently this procedure is able to unveil small differences in atmospheric parameters, removing many sources of systematic errors. As noted by Desidera et al. (2006), external uncertainties due to wrong estimations of parallax and mass, for example, or inadequacies of model atmospheres and LTE deviations, have negligible effects on our results because of the similar characteristics of the components. In the end, the difference in iron abundance of $\mathrm{XO}-2 \mathrm{~N}$ with respect to $\mathrm{XO}-2 \mathrm{~S}$ is of $+0.054 \mathrm{dex}$ at a more than $3 \sigma$ level.

The difference in iron abundance between the two XO-2 stellar components poses an interesting question. They belong to a visual binary and, as normally assumed for such systems, they should share the same origin and initial bulk metallicity. A relevant characteristic of this system is that both of the stars host planets. Thus, for components of wide binaries where at least one star has a planet, a reasonable hypothesis to explain any measured and significant difference in their present-day elemental abundances is that the planet formation process had played a relevant role. The higher iron abundance of XO-2N when compared to XO-2S might be due to past ingestion of dust-rich or rocky material that came from the inner part of the protoplanetary disk and that was pushed into the host star by the hot Jupiter $\mathrm{XO}-2 \mathrm{~N}$ as it migrated inward to its current orbit. A second mechanism, which acts on a different time scale and after the pre-main-sequence stage, is presented by Fabrycky \& Tremaine (2007), who discuss the pollution of the stellar photosphere with metals produced by mass loss of inward migrating hot Jupiters. In addition, Kaib et al. (2013) showed with their simulations that very distant binary companions may severely affect planetary evolution and influence the orbits of any planet around the other component of the system, perhaps favouring the ingestion of material by the host star. Following the results shown in Fig. 2 of Pinsonneault et al. (2001), we roughly estimated that $\mathrm{XO}-2 \mathrm{~N}$ could have ingested an amount of iron slightly higher than $5 M_{\oplus}$ to increase its photospheric iron content by $\sim 0.05 \mathrm{dex}$, given its $T_{\mathrm{eff}} \sim 5300 \mathrm{~K}$. The difference in the iron abundance of the two XO-2 stellar components is similar to 

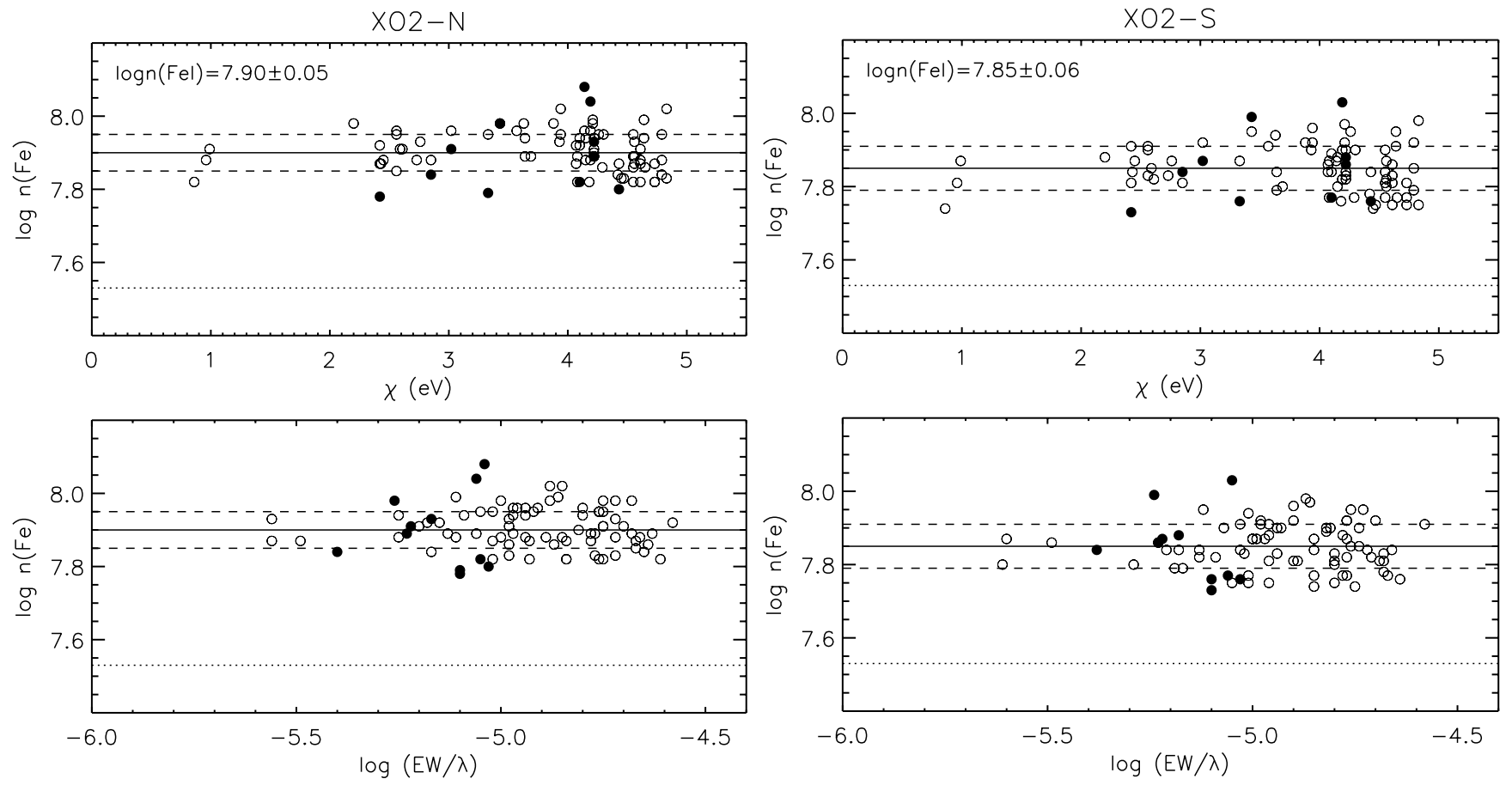

Fig. 2. Iron abundance of the XO-2 stellar binary derived through the analysis based on equivalent widths as a function of the excitation potential and reduced equivalent widths. Empty and filled circles represent the Fe I and Fe II lines, respectively. Solid lines show the location of mean $\log n(\mathrm{Fe} \mathrm{I})$, while dashed lines represent the standard deviation $\pm 1 \sigma$. The dotted line is intended to guide the eye to the solar iron abundance at $\log n(\mathrm{Fe} \mathrm{I})_{\odot}=7.53$.

that found by Ramírez et al. (2011) for the solar twins 16 Cyg A and $16 \mathrm{Cyg} \mathrm{B}(\Delta[\mathrm{Fe} / \mathrm{H}]=0.042 \pm 0.016 \mathrm{dex})$. These stars have masses close to those of the XO-2 companions and, together with a third companion $16 \mathrm{Cyg} \mathrm{C}$, are members of a hierarchical triple system, where the $\mathrm{A}$ and $\mathrm{C}$ components form a close binary with a projected separation $\sim 70$ AU. The component $\mathrm{B}$ is known to host a giant planet in a long-period and highly eccentric orbit $(P \sim 800 \text { days and } e \sim 0.69)^{5}$, but unlike the case of XO-2 it is the star with a lower iron content. To explain this deficiency, Ramírez et al. (2011) suggest that an early depletion of metals happened during the formation phase of the $16 \mathrm{Cyg} \mathrm{Bb}$ planet. An alternative and suggestive possibility is that 16 Cyg A may have ingested a massive planet that enriched the star with iron, while the large orbit of $16 \mathrm{Cyg} \mathrm{Bb}$ (with semi-major axis of $1.68 \mathrm{AU}$ ) very likely prevented any mass loss from the planet. We note that, although $\Delta[\mathrm{Fe} / \mathrm{H}]$ is similar to that found for XO-2, the amount of iron involved should be different. The XO-2 stars are cooler than 16 Cygni A and B and therefore have more massive convective envelopes, implying that more iron should be [is?] necessary to pollute the XO-2N photosphere and produce almost the same $\Delta[\mathrm{Fe} / \mathrm{H}]$. Laws \& Gonzalez (2001) also found the primary of the 16-Cyg system enhanced in Fe relative to the secondary. Similar studies conducted in binary stars hosting planets (see e.g. Gratton et al. 2001; Desidera et al. 2004, 2006; Schuler et al. 2011a; Teske et al. 2013; Liu et al. 2014; Mack et al. 2014) did not find relevant differences in elemental abundance among the components. All these results imply that the presence of giant planets does not necessarily imply differences in the chemical composition of the host star.

Our results will produce a clearer picture when several iron-peak, $\alpha$-, s-process, and other odd/even-Z elemental abundances are analysed for both stars and their behaviour with the

\footnotetext{
5 http://exoplanet.eu
}

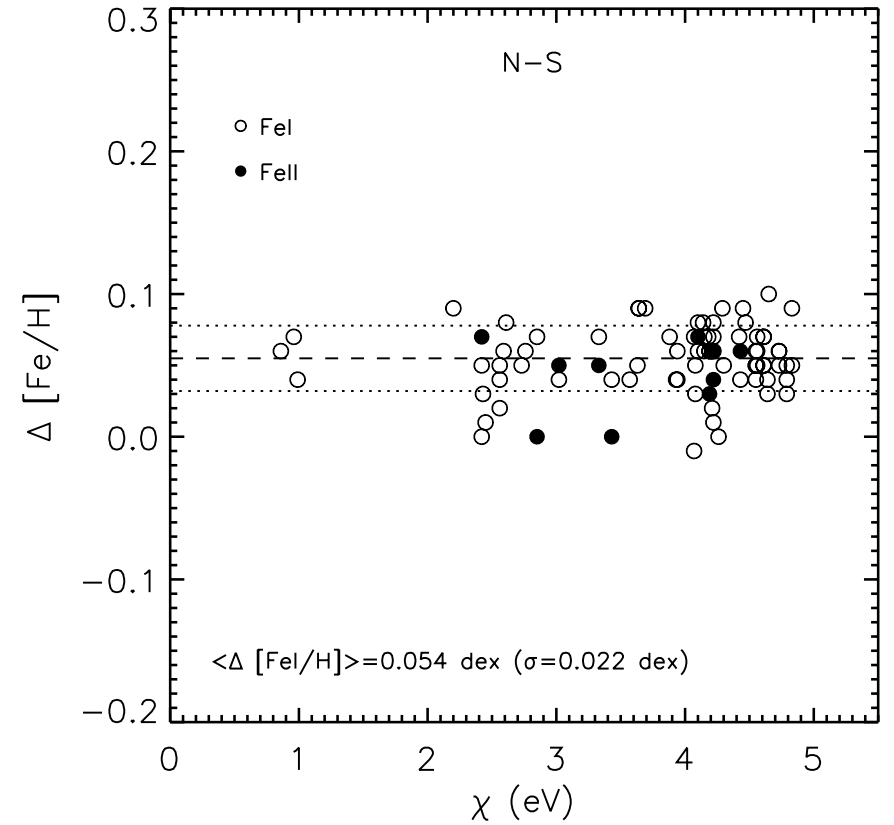

Fig. 3. Iron abundance difference between $\mathrm{XO}-2 \mathrm{~N}$ and $\mathrm{XO}-2 \mathrm{~S}$ vs. $\chi$. Dashed line shows the mean $\Delta[\mathrm{Fe} / \mathrm{H}]$, while dotted lines represent the standard deviation $\pm 1 \sigma$ from the mean, where $\sigma=0.022$ dex.

condensation temperature studied with the aim of investigating possible selective accretion of planetary material. One of the advantages of comparing coeval stars in wide binaries is that any difference in their abundance trend could be more closely related to the accretion of rocky-planetary material rather than to the Galactic chemical evolution. This analysis will be the subject of a forthcoming paper of the GAPS series (Biazzo et al., in prep.), 
where we will investigate if the presence of hot Jupiters could lead the host stars to ingest material, which in turn may leave measurable chemical imprints in their atmospheric abundances.

\subsection{XO-2 distance and galactic space velocities}

Using our stellar parameters, we derived an estimate of the spectroscopic distances of $\mathrm{XO}-2 \mathrm{~N}$ and $\mathrm{XO}-2 \mathrm{~S}$ by means of the following procedure. We generated Monte Carlo (MC) normal distributions for each spectroscopic parameter $T_{\text {eff }},[\mathrm{Fe} / \mathrm{H}]$, and $\log g$, composed of 10000 random values and centred on the best estimates (Table 2). By keeping the stellar radii fixed to the values listed in Table 2 (for $\mathrm{XO}-2 \mathrm{~N}$ we used the most accurate estimate), for each MC simulation we first determined the stellar bolometric luminosity $L_{*}$ (in solar units) from the Stefan-Boltzmann law, and then we derived the absolute bolometric magnitude $M_{\mathrm{bol}}$ from the relation $M_{\mathrm{bol}}=$ $4.75-2.5 \cdot \log \left(L_{*}\right)$. By estimating the appropriate bolometric correction (BC), a value for the absolute magnitude in $V$-band $M_{V}$ was then obtained. The $\mathrm{BC}$ term was evaluated using the code provided by Casagrande \& VandenBerg (2014). An additional input is the colour excess $E(B-V)$ of the star, which we derived through the relation $E(B-V)=A_{V}(\mathrm{~s}) / 3.1$, where $A_{V}(\mathrm{~s})$ is the interstellar dust extinction in $V$-band integrated at the distance $s$ of the star (in pc) and measured along the line of sight. We derived $A_{V}(\mathrm{~s})$ by adopting a simplified model of the local distribution of the interstellar dust density (Drimmel \& Spergel 2001), expressed by the relation $\rho=\rho_{0} \cdot \operatorname{sech}^{2}\left(z / h_{\mathrm{s}}\right)$, where $z$ is the height of the star above the Galactic plane and $h_{\mathrm{s}}$ is the scaleheight of the dust, for which we adopted the value of $190 \mathrm{pc}$. The term $z$ is related to the distance $s$ and the Galactic latitude of the star $b$ by the formula $z=s \cdot \sin b$. From this model we obtained the relation $A_{V}(\mathrm{~s})=A_{V}($ tot $) \cdot \sinh \left(s \cdot \sin b / h_{\mathrm{s}}\right) / \cosh \left(s \cdot \sin b / h_{\mathrm{s}}\right)$, where $A_{V}$ (tot) is the interstellar extinction in $V$ band along the line of sight integrated through the Galaxy, and can be estimated from 2D Galactic maps. For this purpose we used the value $A_{V}$ (tot) $=0.16$ mag derived from the maps of Schlafly \& Finkbeiner (2011) $)^{6}$. By assuming $s=150 \mathrm{pc}$ as a prior distance of the stars (Burke et al. 2007), we obtained $A_{V}(150 \mathrm{pc}) \sim 0.06 \mathrm{mag}$, corresponding to $E(B-V)=0.019 \mathrm{mag}$. This is the value used as input to the code of Casagrande \& VandenBerg (2014) to obtain a first guess of the $\mathrm{BC}$ in $V$-band. This in turn was used in the distance modulus formula $V-\left(M_{\mathrm{bol}}-\mathrm{BC}_{V}\right)=5 \cdot \log (\mathrm{s})$ $-5-\mathrm{A}_{V}(\mathrm{~s})$, to obtain a new value for the stellar distance $s$. The new distance was used to repeat the procedure iteratively, by determining at each step a new value of $A_{V}(\mathrm{~s})$ and $B C_{V}(\mathrm{~s})$, and finally another estimate of $s$. When the absolute difference between the last and previous calculated values of $s$ was below $0.1 \mathrm{pc}$, the iterative process was interrupted and the last derived value for $s$ was assumed as the distance of the star for the Nth Monte Carlo simulation. The adopted estimates for the distance of the XO-2 components are the median of the distributions of the $10000 \mathrm{MC}$ values, and the asymmetric error bars defined as the 15.85th and 68.3th percentile (see Table 1). Because they are model-dependent, we do not argue here whether the difference of $\sim 2.5 \mathrm{pc}$ between the $\mathrm{XO}-2 \mathrm{~S}$ and $\mathrm{XO}-2 \mathrm{~S}$ distances is real. We only note that the two values are compatible within the uncertainties and that our best estimate for the distance of $\mathrm{XO}-2 \mathrm{~N}$ locates the star a few parsec closer than reported by Burke et al. (2007).

\footnotetext{
6 Available at http://irsa.ipac.caltech.edu/applications/ DUST/
}

Distances, together with the stellar equatorial coordinates, the proper motions, and the average radial velocities (Tables 1 and 7), were used to provide new estimates of the galactic space velocities $(\mathrm{U}, \mathrm{V}, \mathrm{W})$ of the stars with respect to the local standard of rest (LSR). We thus assumed (i) a left-handed system of reference (i.e. the velocity component $U$ is positive toward the direction of the Galactic anti-centre); (ii) the transformation matrix from equatorial to galactic coordinates taken from the HIPPARCos catalogue (Perryman \& ESA 1997); and (iii) the correction for the solar motion in the LSR derived from Coşkunoğlu et al. (2011). The results are listed in Table 1. Burke et al. (2007) discussed the kinematics of the XO-2 system by using data for $\mathrm{XO}-2 \mathrm{~N}$ only, and concluded that, despite the high galactic space velocity which indicates a thick disk membership, the supersolar metallicity is indeed representative of a thin disk membership. This suggests that the high-proper motion of the system can be related to an eccentric orbit with a maximum height above the galactic plane of $\sim 100 \mathrm{pc}$, then confined to the galactic disk.

We performed a new analysis of the galactic orbits, based on the work of Barbieri \& Gratton (2002), and we confirm the previous findings. Results for a sample of 1000 orbits show that the XO-2 system belongs to the thin disk, with a likely maximum height above the galactic plane of $120 \mathrm{pc}$. This result does not change significantly unless the distance of the stars is at least 20 pc more or less than our estimates. Our analysis also indicates a possible eccentric orbit, with a modal eccentricity equal to $0.42_{-0.06}^{+0.09}$ (the asymmetric errorbars are defined as the 10th and 90th percentile), and a minimum distance from the galactic centre of $R_{\min }=3.5 \pm 1.5 \mathrm{kpc}$. If we assume $R_{\min }$ as a rough estimate of the galactic region where the two stars formed, this could explain their super-solar metallicity, because regions close to the galactic centre are more metal rich than the solar neighbourhood. Moreover, this could also have modified the orbit of the binary with time because of the influence of the denser stellar environment close to the galactic centre, with important consequences on the evolution of the orbits of the planets.

\section{Analysis of the light curves}

\subsection{Out-of-transit photometry}

Figure 4 shows the relative photometry of the two components of the XO-2 system obtained from different facilities. Measurements were collected in the BVRI standard photometric passbands and the points corresponding to the transits of the planet $\mathrm{XO}-2 \mathrm{Nb}$ were not included in the calculation of the intra-night average values. The uncertainties associated with the measurements are the RMS of the points collected in each single night of observation, and when only one measurement per night was available from SLN, we adopted the dispersion of all the data as the error. The average values for the magnitude differences in each band are indicated in Table 1 . We note that the independent measurements in $R$ (TASTE and SLN) and $I$ (APACHE and SLN) bands agree well with each other. Our derived relative magnitudes have been used in the differential abundance analysis discussed in Sect. 3.2.

On the night between October 31 and November 1, 2014, over a period of $30 \mathrm{~min}$, we took a series of $60 \mathrm{~s}$ exposures of the XO-2 field with the Asiago Faint Object Spectrograph and Camera (AFOSC) at the $182 \mathrm{~cm}$ Asiago Astrophysical Observatory telescope, while the field was close to the zenith. By placing both components in a $8^{\prime \prime}$ slit and using a grism covering the spectral range $3900-8000 \AA$, we obtained a final simultaneous differential spectrum by dividing the combined individual 
M. Damasso et al.: The GAPS programme with HARPS-N at TNG. V.
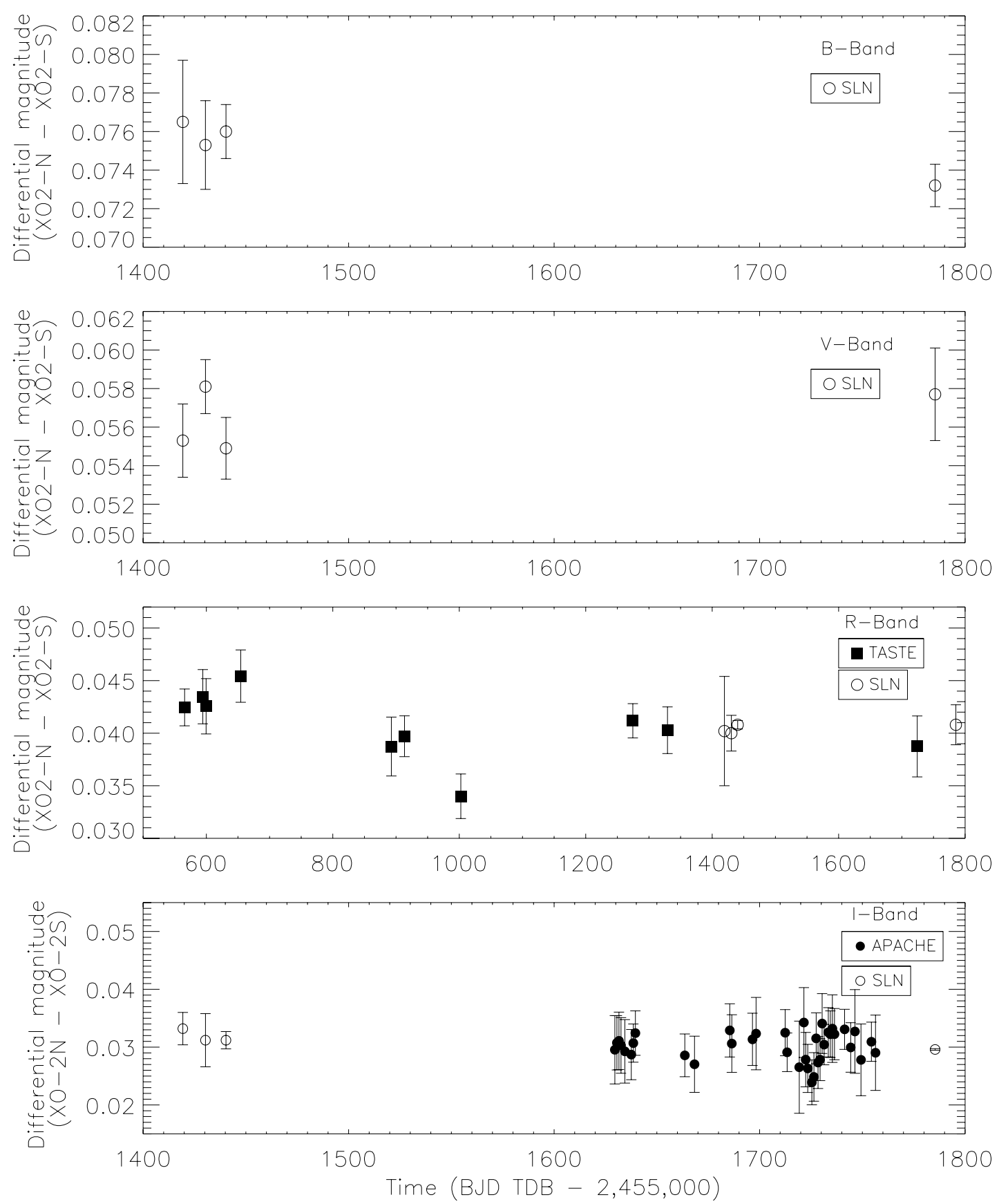

Fig. 4. Magnitude differences between the star XO-2N and its companion XO-2S as measured in BVRI passbands from data of the TASTE project, APACHE survey, and Serra La Nave Observatory. These differences were evaluated from out-of-transit photometry.

spectra in each wavelength resolution element by the other value. The differential spectrum is shown in Fig. 5. These observations were characterized by a very good instrumental stability and strengthened the results obtained with broadband measurements of the differential magnitudes. These agree well with the AFOSC measurements, as shown in Fig. 5. Because of the high scatter of the data in the $I$ band caused by fringing, we did not include in the plot the measurements with wavelengths greater than $\sim 770 \mathrm{~nm}$.

\subsubsection{Rotational periods}

Thanks to the time span of the APACHE $I$ band observations and $\sim 800$ useful data points for each component, we were able to analyse the photometric time series to search for periodic modulations ascribable to the rotation of the stars and produced by brightness inhomogeneities distributed on the stellar photospheres (which are assumed to change on time scales longer than the time span of the observations). Taking into account that the XO-2 system is quite old (see Table 2) and the two stars are magnetically quiet (see discussion in Sect. 6), they are expected to have rotation periods of several tens of days (30-40 days) and low amplitudes in the light curve variations. We used the generalized Lomb-Scargle algorithm (GLS; Zechmeister \& Kürster 2009) to search for periodicities in our photometric time series, after binning the data collected during each night in a single point. The data are available online at the CDS, each containing the timestamps, differential magnitudes, and corresponding 


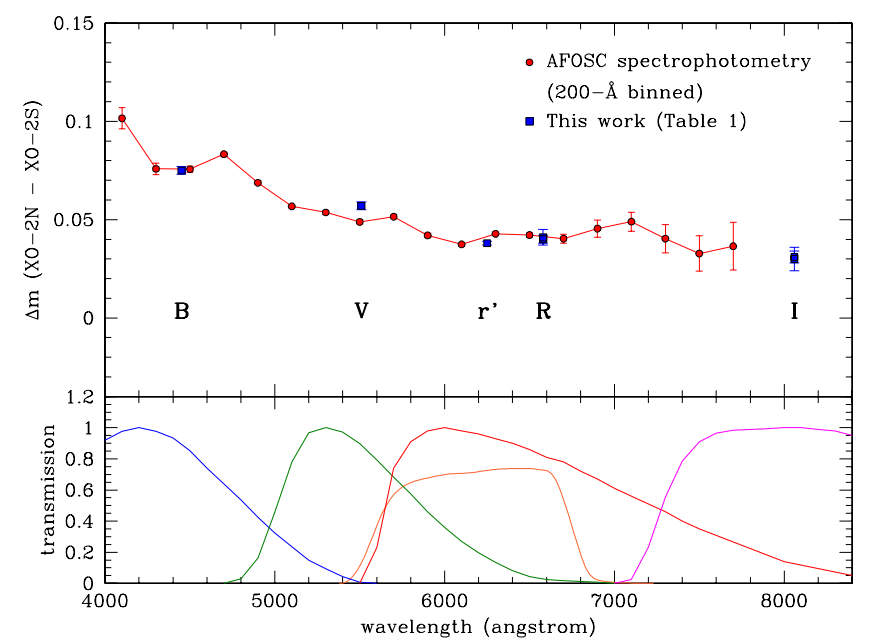

Fig. 5. Upper panel: differential spectro-photometry of the XO-2 components obtained with the AFOSC camera at the $182 \mathrm{~cm}$ Asiago telescope. Red dots represent the mean values of measurements in bins of $200 \AA$, while blue squares indicate the broadband differential magnitudes presented in Table 1. Lower panel: bandpasses of the filters used for the broadband measurements.
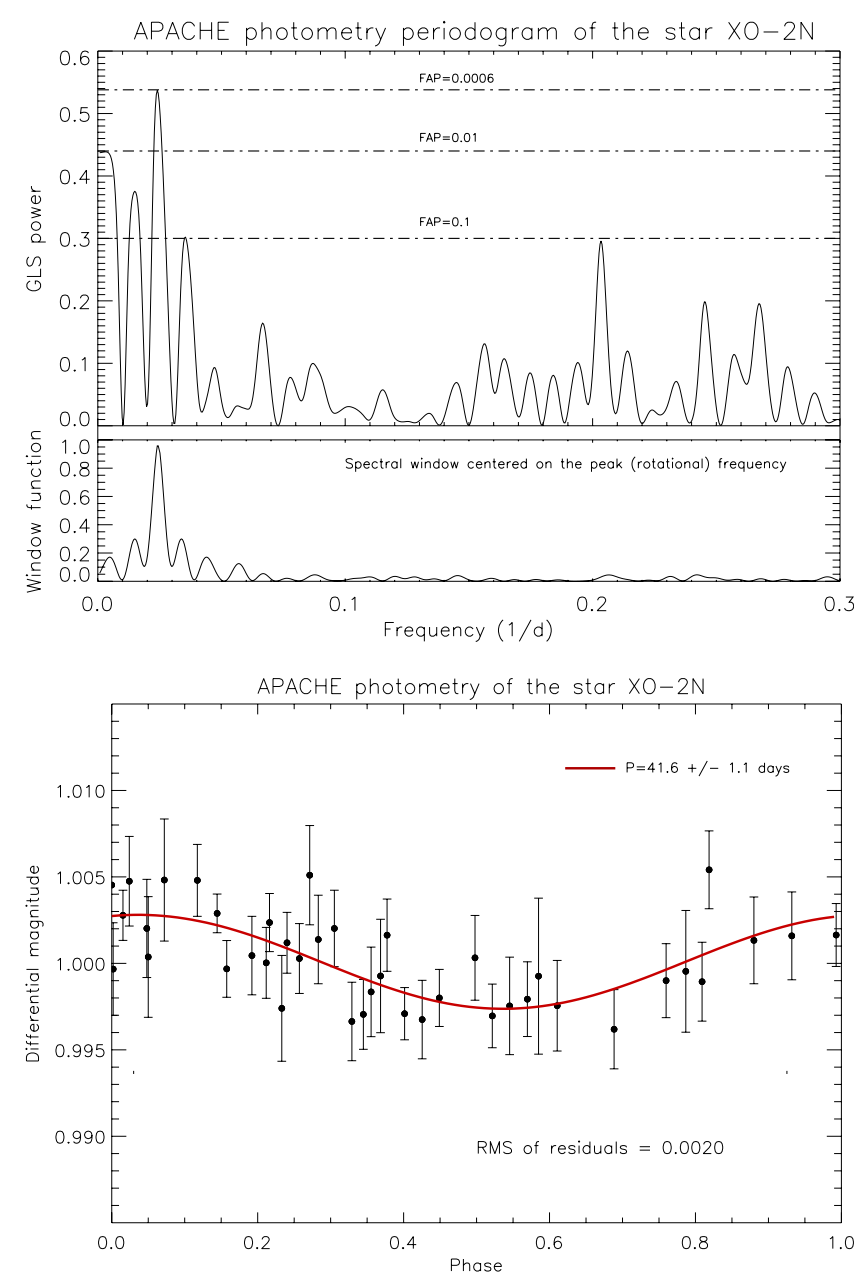

Fig. 6. Upper panel: the GLS periodogram of the XO-2N light curve collected by the APACHE survey and the corresponding window function, with the highest peak translated to the frequency of the best significant peak found in the periodogram. Levels of different FAP are also indicated. Lower panel: the APACHE light curve of XO-2N folded at the best period found by GLS with superposed the best sinusoidal fit of semi-amplitude $A=0.0027 \mathrm{mag}$ (solid red line).
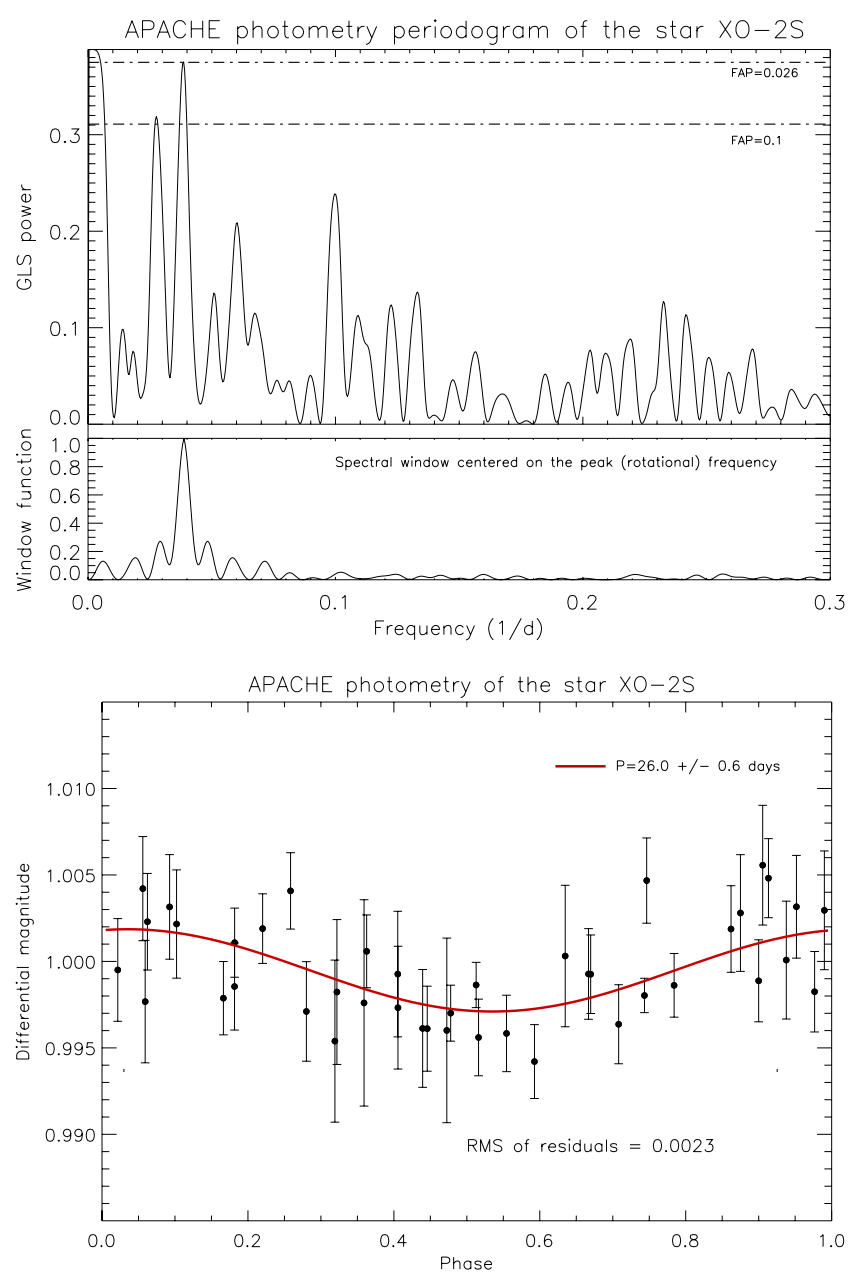

Fig. 7. Upper panel: GLS periodogram of the XO-2S light curve collected by the APACHE survey and the corresponding window function, with the highest peak translated to the frequency of the best significant peak found in the periodogram. Levels of different FAP are also indicated. Lower panel: APACHE light curve of XO-2S folded at the best period found by GLS with superposed the best sinusoidal fit of semiamplitude $A=0.0024 \mathrm{mag}$ (solid red line).

uncertainties. Results are shown in Figs. 6 and 7 for XO-2N and $\mathrm{XO}-2 \mathrm{~S}$. The major peaks at low frequencies in the periodograms are due to the limited time baseline of our observations. For the northern component we find a peak in the periodogram corresponding to a period $P=41.6 \pm 1.1$ days, with a semi-amplitude of the folded light curve of $0.0027 \pm 0.0004 \mathrm{mag}$. Because there is no evidence of harmonics in the periodogram, the measurements can be arranged almost perfectly with a sine function. To estimate the significance of the best period, we performed a bootstrap analysis (with replacement) by using 10000 fake datasets derived from the original photometry, and in six cases we found a power greater than that associated with the best period in the real dataset. This corresponds to a false alarm probability (FAP) equal to $0.06 \%$, which makes the result for $\mathrm{XO}-2 \mathrm{~N}$ rather robust.

Interestingly, to our knowledge two other systems with an old star have measured rotation periods thanks to Kepler data. The A and B components of the 16 Cygni system, which is estimated to be $6.87 \pm 2 \mathrm{Gyr}$ old, have rotation periods $23.8_{-1.8}^{+1.5}$ and $23.2_{-3.2}^{+11.5}$ days, respectively (Davies et al. 2015). Another example is that of Kepler-77, a star-planet system $7 \pm 2$ Gyr old. The detected stellar rotation period is $36 \pm 6$ days, with sizeable 
changes in the shape of the light curve modulation (Gandolfi et al. 2013). This case, and especially that of 16 Cyg B with uncertainties of several days, demonstrate that for old late-type stars the determination of the true rotation period can be particularly difficult. The intrinsic evolution of their surface active regions takes place on a time scale shorter than the rotation period, and therefore starspots become bad tracers of stellar rotation. In the Sun, depending on the phase of the solar cycle, the rotation period measured by fitting a sinusoid to the total solar irradiance - a good proxy for the Sun-as-a-star optical modulation varies between 22.0 and 31.9 days (the true synodic period being 27.27 days). The variations are especially large during the maximum of the solar cycle because several large active regions are simultaneously present on the solar surface and evolve on time scales of 1-2 weeks, i.e. significantly shorter than the true rotation period (e.g. Lanza et al. 2003).

Even if the rotation period of XO-2N appears well constrained, the complications expected for old late-type stars could actually have prevented us from detecting an accurate value. This is indeed the case for the companion star XO-2S, for which a less convincing detection resulted in our data. The GLS periodogram returned the highest peak signal at $f=0.0384$ days $^{-1}$, corresponding to $P=26.0 \pm 0.6$ days, with a semi-amplitude of $0.0024 \pm 0.0005 \mathrm{mag}$ of the folded light curve. For XO-2S there is also no evidence of harmonics. The bootstrap analysis in this case led to a FAP $=2.6 \%$, which casts some doubts on the reality of the signal, even if the period is still compatible with the measured projected velocity $V \sin I_{\star}$. The similarity between the observed periodogram and the recentred spectral window (bottom graph in the upper panel of Fig. 7) strengthens our confidence on the stellar origin of the periodicity we found. However, a second peak at 0.029 days $^{-1}(P=34.5$ days $)$ with a slightly less power must be noted. This has some relevance in the discussion, since a rotational period of 34.5 days matches the gyrochronology relations (e.g. see Fig. 13 in Barnes 2007) better than the one of 26 days, and it is also closer to the estimates based on measurements of the activity index $R_{\mathrm{HK}}^{\prime}$ (see Table 8). Moreover, in analogy with the case of the Sun-as-a-star, if we assume $P=34.5$ days as the best detection, it is reliable that the star has a rotation period a bit higher than that. Therefore more data are necessary, possibly covering several activity cycles, to reach a more robust conclusion concerning the true rotational period of XO-2S.

\subsection{Transit photometry of $X \mathrm{O}-2 \mathrm{~N}$}

Following the discovery of the hot Jupiter $\mathrm{XO}-2 \mathrm{Nb}$ (Burke et al. 2007), new transit light curves of XO-2N were collected to improve the stellar and planetary parameters and to search for transit timing variations (Fernandez et al. 2009; Kundurthy et al. 2013; Crouzet et al. 2012), and the planet atmosphere was analysed with space-based (Machalek et al. 2009; Crouzet et al. 2012) and ground-based observations (Sing et al. 2011, 2012).

Through the combined analysis of 14 new transit light curves, we present here refined parameters for the XO-2N system, together with those in Table 2. The individual transit light curves are shown in Fig. 8, and the full dataset combined in a single, high-quality transit light curve is shown in Fig. 9. The information about each dataset is summarized in Table 4. The raw scientific frames were first corrected for bias, dark and flat-field using standard software tools. The STARSKY pipeline (see Nascimbeni et al. 2013, and references therein) was employed to perform differential photometry between $\mathrm{XO}-2 \mathrm{~N}$ and a set of optimally-weighted reference stars. The whole set of fourteen light curves was then simultaneously analysed with the JKTEBOP ${ }^{7}$ code (version 34, Southworth et al. 2004). The adopted transit model parametrizes the stellar limb darkening (LD) with a quadratic law, i.e. with the two parameters $u_{1}$ and $u_{2}$. Assuming a circular orbit, seven parameters were left free to vary in the fitting procedure: the period $P$ and reference time $T_{0}$ of the underlying transit ephemeris, the ratio $k=R_{\mathrm{p}} / R_{\star}$ and sum $\left(R_{\mathrm{p}}+R_{\star}\right) / a$ of the fractional radii ( $a$ being the semimajor axis of the system), the orbital inclination $i$, and the LD parameters $u_{1}$ and $u_{2}$. Leaving both $u_{1}$ and $u_{2}$ free to vary allows us to avoid putting any a priori assumption on the stellar atmospheric parameters. Our estimates are in good agreement with those derived by interpolating the theoretical tables of Claret \& Bloemen (2011) using our derived stellar atmospheric parameters $\left(0.48\right.$ and 0.21 for $u_{1}$ and $\left.u_{2}\right)$. The transit model was fitted to our data with a non-linear least-squares technique; the uncertainties over the best-fit parameters are then recovered with a bootstrap algorithm, described by Southworth (2008). Other quantities of interest, such as the impact parameter $b$ or the transit duration $T_{14}$, were derived through their analytical expressions from the fitted parameters. The final best-fit values, along with their estimated errors, are summarized in Table 5. We give the estimates as the median of the bootstrapped distributions, with the asymmetric errorbars defined as the 15.87th and 84.13th percentile. Our results are in good agreement with those in the literature, in particular with the results of Crouzet et al. (2012) based on observations made with the Hubble Space Telescope. We were able to provide an updated ephemeris (with an error on $P$ of about $0.025 \mathrm{~s}$ ) that we also used as a prior for the radial velocity fit (Sect. 5). Our determination of the stellar density $\rho / \rho_{\odot}$, thanks to $u_{1}$ and $u_{2}$ being left free to vary, is completely independent from stellar models, and was used as input to interpolate the Y-Y evolutionary tracks and to derive an accurate estimate for the stellar mass, radius, and age (Sect. 3).

\section{Radial velocities of $\mathrm{XO}-2 \mathrm{~N}$}

We observed the star XO-2N as part of a programme aimed at searching for additional outer planets in the system, and we investigated the Rossiter-McLaughlin (RML) effect by acquiring high-resolution spectra with HARPS-N during one transit event of the planet $\mathrm{XO}-2 \mathrm{Nb}$.

\subsection{Analysis of the radial velocity time series}

To refine the orbital parameters of XO-2N b, our new HARPS-N data were analysed along with the RVs obtained with the High Dispersion Spectrograph (HDS) at the Subaru telescope (Narita et al. 2011), and the High Resolution Echelle Spectrograph (HIRES) at the Keck telescope (Knutson et al. 2014). The original spectroscopic data from Burke et al. (2007) were not included because they have considerably larger error bars $\left(>20 \mathrm{~m} \mathrm{~s}^{-1}\right)$ than the other datasets and thus do not yield any significant improvement of the orbital solution. We first fitted separately the HDS, HIRES, and HARPS-N RV time series with a Keplerian orbit and a slope, after removing the intransit points that are affected by the Rossiter-McLaughlin effect. We then performed a Bayesian analysis with our Differential Evolution Markov chain Monte Carlo (DE-MCMC) tool (see e.g. Desidera et al. 2014). Narita et al. (2011) and Knutson et al. (2014) reported on a positive acceleration revealed in their RV datasets, equal to $0.0206 \pm 0.0016$ and $0.0126_{-0.0036}^{+0.0039} \mathrm{~m} \mathrm{~s}^{-1} \mathrm{day}^{-1}$,

http://www. astro.keele.ac.uk/jkt/codes/jktebop.html 


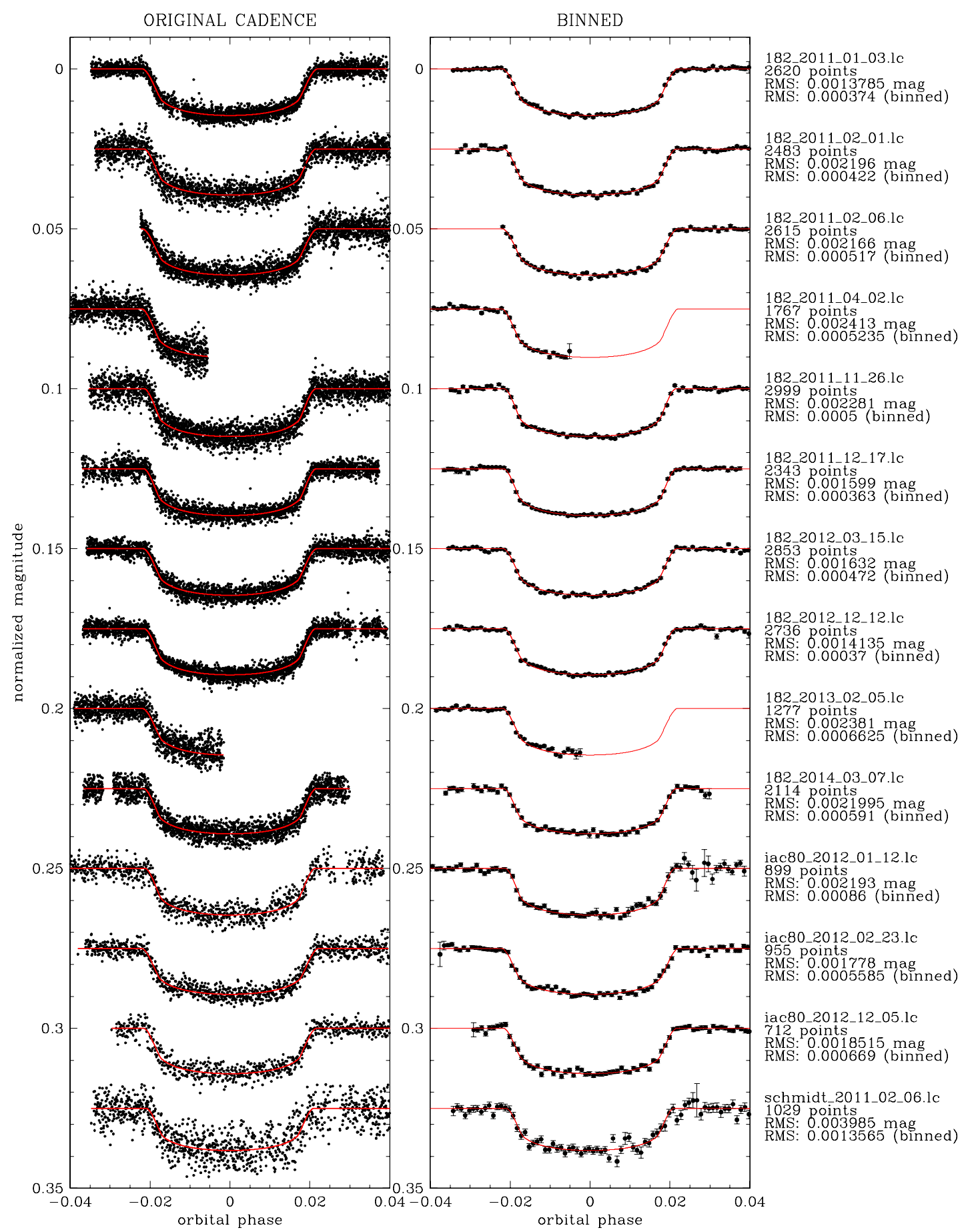

Fig. 8. Transit light curves of the planet XO-2Nb collected with the Copernico $(182 \mathrm{~cm}$ ) and Schmidt $67 / 91 \mathrm{~cm}$ telescopes (Asiago Observatory) within the framework of the TASTE Project (first ten plots and the last one) and, independently, at the IAC-80 telescope (Teide Observatory, Canary Islands; remaining plots). All these observations were carried out in the Cousin/Bessell $R$-band. The light curves are plotted both in their original cadence (left diagrams), and binned over 2 min intervals (right diagrams). For each transit, the RMS of the residuals from the best-fit model is also tabulated. A composite light curve obtained from all the individual time series is used to determine the transit parameters shown in Table 5 , as discussed in the text.

respectively, possibly due to a second massive companion in an outer orbit, namely XO-2N c. Our observations and analysis confirm the existence of this trend, but we observed a turnover in the HARPS-N data and measured a negative acceleration equal to $-0.0170_{-0.0031}^{+0.0033} \mathrm{~m} \mathrm{~s}^{-1} \mathrm{day}^{-1}$.

We then modelled the RV data of the three datasets with a Keplerian orbit and a polynomial model following the formalism of Kipping et al. (2011) and choosing as $t_{\text {pivot }}$ in their
Eq. (1) the mean of the epochs of the RV observations. In total, our RV model has thirteen free parameters: the transit epoch $T_{\mathrm{c}}$; the orbital period $P$; the radial-velocity semiamplitude $K$; $e \cos \omega$ and $e \sin \omega$ of $\mathrm{XO}-2 \mathrm{Nb}$, where $e$ and $\omega$ are the eccentricity and argument of periastron; three systemic velocities $\left(\gamma_{\mathrm{HDS}}, \gamma_{\mathrm{HIRES}}, \gamma_{\mathrm{HARPS}-\mathrm{N}}\right)$ and RV jitter terms $\left(\right.$ jitter $_{\mathrm{HDS}}$, jitter $r_{\text {HIRES }}$, jitter $\left.r_{\text {HARPS-N}}\right)$ corresponding to the three different datasets; and the linear $\dot{\gamma}$ and quadratic $\ddot{\gamma}$ trends. Gaussian priors 


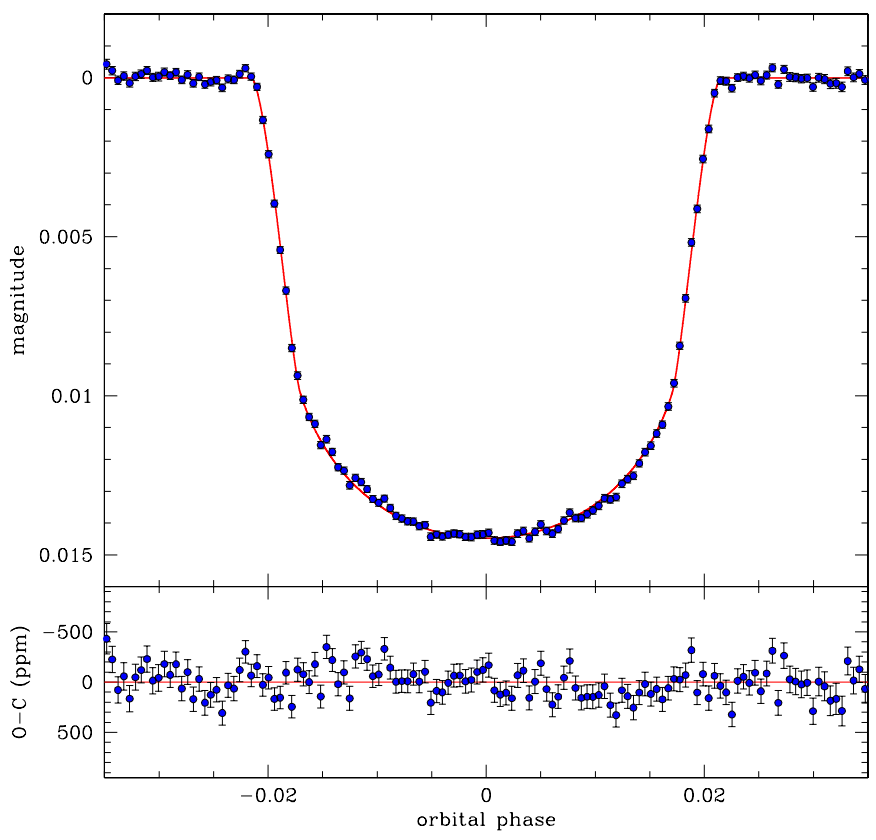

Fig. 9. Photometric data presented in Fig. 8 and combined together in a single transit light curve to appreciate the quality of the whole dataset. Data points are the weighted mean of the measurements in bins of $120 \mathrm{~s}$, and the best-fit transit model is over plotted as a solid line, with the $\mathrm{O}-\mathrm{C}$ residuals shown in the lower panel.

were imposed on i) $T_{\mathrm{c}}$ and $P$ from the new ephemeris we derived in Sect. 4; and ii) the centre times of the secondary eclipse observed by Machalek et al. (2009) at 3.6, 4.5, 5.8, and $8.0 \mu \mathrm{m}$ with Spitzer, because these provide strong constraints on the orbital eccentricity; for this purpose, we used Eq. (18) in Jordán $\&$ Bakos (2008). We adopted Jeffrey's priors for the three jitter terms (see e.g. Gregory 2005) and non-informative priors for the RV semi-amplitude and the three systemic velocities.

After the earliest analysis, we noticed that the first HIRES point at 2454372.14 BJD $_{\text {UTC }}$ was $3 \sigma$ below our best fit hence we discarded it and repeated the analysis, although its inclusion has a very minor influence on the final solution. The posterior distributions of the fitted and derived parameters were obtained with the same procedure as described in Desidera et al. (2014). Their medians and $68.3 \%$ confidence intervals are listed in Table 6, along with the system parameters of XO-2S derived by Desidera et al. (2014). The Keplerian best fit to the three datasets, after removing the quadratic trend, is shown in Fig. 10. Our new RV semi-amplitude $K=89.78 \pm 0.81 \mathrm{~m} \mathrm{~s}^{-1}$ is slightly lower than the value found by Knutson et al. (2014), which is $K=93.9 \pm 2.2 \mathrm{~m} \mathrm{~s}^{-1}$. Its precision is improved by a factor of three, especially thanks to the HARPS-N data. The orbital eccentricity is consistent with zero, $e<0.006$ at $1 \sigma$, which justifies the use of a circular model for the transit modelling (Sect. 4.2). The combined analysis of the complete RV dataset and of the new transit light curves allows us to refine the physical parameters of the planet $\mathrm{XO}-2 \mathrm{Nb}$, and we find that the best values for the radius, mass, and density are $1.019 \pm 0.031 R_{\mathrm{Jup}}$, $0.597 \pm 0.021 M_{\mathrm{Jup}}$, and $0.70_{-0.064}^{+0.072} \mathrm{~g} \mathrm{~cm}^{-3}$, respectively.

\subsubsection{RV acceleration in XO-2N: evidence for an outer companion or solar-like stellar activity cycle?}

Under the hypothesis that an outer massive companion exists and is responsible for the observed acceleration, the change of sign derived from HARPS-N data with respect to the previous measurements could be likely caused by the curvature of the orbit of $\mathrm{XO}-2 \mathrm{~N} \mathrm{c}$. Figure 11 shows the quadratic trend in the RV residuals, after removing the orbital signal of $\mathrm{XO}-2 \mathrm{Nb}$ and considering all the available datasets. By assuming a circular orbit for the outer companion $\mathrm{XO}-2 \mathrm{~N} \mathrm{c}$, the derived quadratic trend allows us to estimate its orbital period and minimum mass (see Kipping et al. 2011): $P \geq 17 \mathrm{yrs}$ and $M \sin i \geq 1.8 M_{\text {Jup }}$. Direct imaging observations collected with the AstraLux instrument did not reveal a companion to $\mathrm{XO}-2 \mathrm{~N}$ with $\Delta i^{\prime} \lesssim 8$ mag and a separation $\lesssim 2 "$ (Daemgen et al. 2009), which corresponds to $\sim 300 \mathrm{AU}$ at the star location. A rough estimate for an upper limit of the $\mathrm{XO}-2 \mathrm{~N} \mathrm{c}$ mass can be made using the information on $\Delta i^{\prime}$. By fixing the age and the metallicity to the values of $\mathrm{XO}-2 \mathrm{~N}$, and using the $\mathrm{CMD}^{8}$ stellar isochrones, we find that the unseen companion should have a mass $M \lesssim 0.16 M_{\odot}$, which roughly corresponds to a main-sequence star of spectral type later than M5V-M6V.

If not due to an outer companion, the quadratic trend could reflect a solar-like activity cycle, which leaves an imprint in the $\mathrm{RV}$ measurements over a time span of several years. This is consistent with the observation that older stars, with longer rotation periods, have longer activity cycles (e.g. Noyes et al. 1984; Ossendrijver 1997). In their work, Knutson et al. (2014) do not mention for $\mathrm{XO}-2 \mathrm{~N}$ the existence of a correlation between their RV residuals and some indicators of the stellar activity, such as $R_{\mathrm{HK}}^{\prime}$ or $S_{\mathrm{HK}}$, which would call the planetary origin of the acceleration into question. The same situation does not occur in our dataset. The time series of the $R_{\mathrm{HK}}^{\prime}$ activity index, calculated according to the recipe described in Lovis et al. (2011), shows the existence of a negative trend (see Fig. 12). We modelled the data with a linear fit, obtaining a negative slope with a $1 \sigma$ uncertainty of $\sim 14 \%$. The Spearman's rank correlation coefficient of the data is quite high $(\rho=-0.79)$ and with a low FAP $\left(1.3 \times 10^{-6}\right)^{9}$, thus strengthening the real existence of a correlation. The direction of the trend being similar for the RV residuals and $R_{\mathrm{HK}}^{\prime}$, a significant positive correlation is observed between them (see Fig. 13), characterized by a Spearman's rank correlation coefficient $\rho=+0.68\left(\mathrm{FAP}=1 \times 10^{-4}\right)$ for $N=26$ measurements.

Because of the importance of this result, the significance of the correlation was further investigated by applying two different tests. Through the first method, described in Figueira et al. (2014, and references therein) which we call the null hypothesis approach, we evaluated the probability of having a Spearman's rank correlation coefficient larger than or equal to that of the real data by testing the null hypothesis, i.e. by assuming that the data pairs are actually uncorrelated. This method, which does not take into account the data uncertainties, employs a Fisher-Yates data shuffling to generate fake data pairs that are uncorrelated. We generated 10000 such random datasets and then calculated the $z$-score for the correlation coefficient of the original dataset relative to the distribution of the simulated Spearman's rank correlation coefficients. The corresponding probability (the $p$-value) is calculated as the one-sided likelihood of having such a $z$-score from the observed Gaussian distribution. The second method, which instead takes into account the data uncertainties, is described in Curran (2014) (called the composite method) and it employs a double Monte Carlo re-sampling of the dataset pairs. First, fake data pairs are generated through a bootstrap method (with re-sampling), with new data pairs randomly chosen from the original ones (i.e. each new pair comes from the real dataset and may appear more than once or not at all in a simulated

\footnotetext{
8 http://stev.oapd.inaf.it/cgi-bin/cmd

9 Values obtained with the R_CORRELATE function in IDL.
} 
Table 4. $\mathrm{Log}$ of the transit observations of $\mathrm{XO}-2 \mathrm{Nb}$ analysed in this work (14 distinct light curves).

\begin{tabular}{lllccc}
\hline \hline Date & Observatory & Telescope/instrument & $N$ & $\begin{array}{c}\sigma \\
(\mathrm{mmag})\end{array}$ & $\begin{array}{c}\sigma_{120 \mathrm{~s}} \\
(\mathrm{mmag})\end{array}$ \\
\hline 2011 Jan. 03 & Asiago & 182 cm/AFOSC & 2620 & 1.37 & 0.37 \\
2011 Feb. 01 & Asiago & 182 cm/AFOSC & 2483 & 2.19 & 0.42 \\
2011 Feb. 06 & Asiago & 67/91 cm Schmidt & 1029 & 3.98 & 1.35 \\
2011 Feb. 06 & Asiago & 182 cm/AFOSC & 2615 & 2.16 & 0.51 \\
2011 Apr. 02 & Asiago & 182 cm/AFOSC & 1767 & 2.41 & 0.52 \\
2011 Nov. 26 & Asiago & 182 cm/AFOSC & 2999 & 2.28 & 0.50 \\
2011 Dec. 17 & Asiago & 182 cm/AFOSC & 2343 & 1.59 & 0.36 \\
2012 Jan. 12 & Teide & IAC80/CAMELOT & 899 & 2.19 & 0.86 \\
2012 Feb. 23 & Teide & IAC80/CAMELOT & 955 & 1.77 & 0.55 \\
2012 Mar. 15 & Asiago & 182 cm/AFOSC & 2853 & 1.63 & 0.47 \\
2012 Dec. 05 & Teide & IAC80/TCP & 712 & 1.85 & 0.66 \\
2012 Dec. 12 & Asiago & 182 cm/AFOSC & 2736 & 1.41 & 0.37 \\
2013 Feb. 05 & Asiago & 182 cm/AFOSC & 1277 & 2.38 & 0.66 \\
2014 Mar. 07 & Asiago & 182 cm/AFOSC & 2114 & 2.19 & 0.59 \\
\hline
\end{tabular}

Notes. All of them were obtained using a Cousin/Bessell $R$ filter. The columns list: the evening date, the observatory and instrument employed, the number of data points $N$, the photometric rms of the residuals from the best-fit model (both for the original cadence, $\sigma$, and after binning over $120 \mathrm{~s}$ intervals, $\left.\sigma_{120}\right)$.

dataset). The second step consists in randomly shuffling each measurement within its uncertainty following a normal distribution of width 1 and centred on 0 . These two steps are repeated 10000 times, resulting in a distribution of Spearman's rank correlation coefficients for which the mean and the standard deviation are calculated. Moreover, the $z$-score is calculated for each simulated dataset through the Fisher transformation of the correlation coefficient, as discussed in Curran (2014), resulting in a $z$-score distribution for which the mean and the standard deviation are provided. The $z$-score defines the level of significance of a correlation, being the number of $\sigma$ from a null correlation for the original dataset.

The null hypothesis test applied to the RV residuals and $R_{\mathrm{HK}}^{\prime}$ dataset for $\mathrm{XO}-2 \mathrm{~N}$ resulted in $z$-score $=-3.41$, corresponding to $p$-value $=0.032 \%$, while the composite method resulted in $\rho_{\text {Spearman }}=0.54 \pm 0.15$ and $z$-score $=1.267 \pm 0.451$. Both results suggest that the null hypothesis can be rejected for our dataset.

A linear regression applied to the same dataset results in a slope of $30.2 \mathrm{~m} \mathrm{~s}^{-1}$ per unit of $R_{\mathrm{HK}}^{\prime}$, with a $1 \sigma$ uncertainty of $\sim 10 \%$. Comparing this slope value with that predicted by the empirical model described by Eq. (13) in Lovis et al. (2011) for an inactive star with the same $T_{\text {eff }}$ and $[\mathrm{Fe} / \mathrm{H}]$ of $\mathrm{XO}-2 \mathrm{~N}$, i.e. $32.9 \pm 0.2 \mathrm{~m} \mathrm{~s}^{-1}$ per unit of $R_{\mathrm{HK}}^{\prime}$, we find that our estimate is in agreement with that expected for RV variations produced by a long-term stellar activity cycle.

The positive correlation found between the RV residuals and $R_{\mathrm{HK}}^{\prime}$ indicates that the variations seen in the RV residuals are likely related to the intrinsic behaviour of the star. During a phase of high stellar activity a net redshift should appear in the RV measurements as a consequence of the suppressed convective motion in the photospheric active regions, which gives a blueshift contribution (see e.g. Dumusque et al. 2012 and references therein). This means that a negative trend in the RVs is expected when a star is observed during a declining phase of its stellar cycle, with a similar trend appearing in the $R_{\mathrm{HK}}^{\prime}$ index. Although there is evidence in our data in favour of the stellar activity cycle as the cause of the RV variations over nearly 2 years, in our opinion the covered time span is not yet sufficiently long to corroborate this conclusion and to exclude the outer massive companion as an alternative explanation. More data are
Table 5. Transit parameters derived from the combined analysis of the 14 transit light curves of $\mathrm{XO}-2 \mathrm{Nb}$ plotted in Fig. 8.

\begin{tabular}{ll}
\hline \hline Parameter & Value \\
\hline$P(\mathrm{~d})$ & $2.61585922 \pm 0.00000028$ \\
$\left(R_{\mathrm{p}}+R_{\star}\right) / a$ & $0.1393 \pm 0.0018$ \\
$R_{\mathrm{p}} / R_{\star}$ & $0.10490_{-0.00063}^{+0.00059}$ \\
$\left(R_{\mathrm{p}} / R_{\star}\right)^{2}$ & $0.01100 \pm 0.00013$ \\
$i(\mathrm{deg})$ & $87.96_{-0.34}^{+0.42}$ \\
$a / R_{\star}$ & $7.928_{-0.093}^{+0.099}$ \\
$b$ & $0.287_{-0.055}^{+0.043}$ \\
$T_{14}(\min )$ & $162.15 \pm 0.36$ \\
$T_{14}(\mathrm{~d})$ & $0.11260 \pm 0.00025$ \\
$T_{c}(\mathrm{BJD} \mathrm{TDB}-2450000)$ & $5565.546480 \pm 0.000054$ \\
$u_{1}$ & $0.474_{-0.028}^{+0.030}$ \\
$u_{2}$ & $0.171_{-0.070}^{+0.067}$ \\
$\rho / \rho_{\odot}$ & $0.960_{-0.034}^{+0.037}$ \\
\hline
\end{tabular}

Notes. The listed parameters are orbital period $P$, sum and ratio of the fractional radii $\left(R_{\mathrm{p}}+R_{\star}\right) / a,\left(R_{\mathrm{p}} / R_{\star}\right)$, orbital inclination $i$, semi-major axis $a$ scaled by stellar radius $R_{\star}$, impact parameter $b$, total duration of the transit from the first to the fourth contact $T_{14}$, reference time of the ephemeris $T_{0}$ (time standard is barycentric Julian Date calculated from UTC times: BJD $\mathrm{DTC}_{\mathrm{UT}}$ ), the linear and quadratic LD parameters $u_{1}$ and $u_{2}$, the average stellar density $\rho / \rho_{\odot}$.

necessary over a longer time baseline to cover at least one activity cycle of $\mathrm{XO}-2 \mathrm{~N}$ and to investigate in greater detail the correlation with the RV measurements.

\subsection{The Rossiter-McLaughlin effect}

Dedicated observations of the RML effect for the XO-2N system were carried out with HARPS-N during the transit which occurred on December 4-5, 2012. The RML observations for this system were previously performed by Narita et al. (2011), who concluded that the orbit of $\mathrm{XO}-2 \mathrm{Nb}$ is unlikely to be highly 
M. Damasso et al.: The GAPS programme with HARPS-N at TNG. V.

Table 6. Orbital parameters for the XO-2N and XO-2S systems. Errors and upper limits refer to $1 \sigma$ uncertainties.

\begin{tabular}{|c|c|c|c|c|c|}
\hline Parameter & $\mathrm{XO}-2 \mathrm{Nb}$ & $\mathrm{XO}-2 \mathrm{Nc}^{*}$ & $\mathrm{XO}-2 \mathrm{Sb}$ & $\mathrm{XO}-2 \mathrm{Sc}$ & Note \\
\hline $\mathrm{P}$ [days] & $2.61585922 \pm 2.8 \times 10^{-7}$ & $\geq 17$ years & $18.157 \pm 0.034$ & $120.80 \pm 0.34$ & fitted \\
\hline $\mathrm{K}\left[\mathrm{m} \mathrm{s}^{-1}\right]$ & $90.17 \pm 0.82$ & $\geq 20$ & $20.64 \pm 0.85$ & $57.68 \pm 0.69$ & fitted \\
\hline$K \cdot P^{-2}\left[\mathrm{~m} \mathrm{~s}^{-1}\right.$ day $\left.^{-2}\right]$ & - & $-5.13 \pm 0.54 \times 10^{-7}$ & - & - & derived \\
\hline$\sqrt{e} \sin \omega$ & - & - & $-0.314_{-0.052}^{+0.059}$ & $-0.388_{-0.012}^{+0.013}$ & fitted \\
\hline$\sqrt{e} \cos \omega$ & - & - & $0.282_{-0.065}^{+0.054}$ & $-0.038_{-0.034}^{+0.033}$ & fitted \\
\hline$e \cos \omega$ & $-0.0009_{-0.0022}^{+0.0034}$ & - & - & - & fitted \\
\hline$e \sin \omega$ & $-0.0004_{-0.0061}^{+0.0040}$ & - & - & - & fitted \\
\hline$e$ & $<0.006$ & - & $0.180 \pm 0.035$ & $0.1528_{-0.0098}^{+0.0094}$ & derived \\
\hline$\omega[\mathrm{deg}]$ & unconstrained & - & $311.9 \pm 9.5$ & $264.5 \pm 4.9$ & derived \\
\hline$T_{\mathrm{c}}\left[\mathrm{BJD}_{\mathrm{TDB}}-2450000\right]$ & $5565.546480 \pm 5.4 \times 10^{-5}$ & - & $6413.11_{-0.86}^{+0.82}$ & $6408.1_{-1.9}^{+1.8}$ & fitted \\
\hline$\gamma_{\mathrm{HDS}}\left[\mathrm{ms}^{-1}\right]$ & $-17.3 \pm 2.0$ & - & - & - & fitted \\
\hline$\gamma_{\text {HIRES }}\left[\mathrm{m} \mathrm{s}^{-1}\right]$ & $-13.5 \pm 2.9$ & - & - & - & fitted \\
\hline$\gamma_{\text {HARPS-N }}\left[\mathrm{m} \mathrm{s}^{-1}\right]$ & $46932.9 \pm 1.7$ & - & \multicolumn{2}{|c|}{$46543 \pm 1$} & fitted \\
\hline jitter $_{\mathrm{HDS}}\left[\mathrm{m} \mathrm{s}^{-1}\right]$ & $<1.2$ & - & - & - & fitted \\
\hline$j i t t e r_{\mathrm{HIRES}}\left[\mathrm{m} \mathrm{s}^{-1}\right]$ & $7.0_{-2.0}^{+2.9}$ & - & - & - & fitted \\
\hline jitter $_{\text {HARPS-N }}\left[\mathrm{m} \mathrm{s}^{-1}\right]$ & $2.6 \pm 0.7$ & - & \multicolumn{2}{|c|}{$1.80 \pm 0.43$} & fitted \\
\hline$\dot{\gamma}\left[\mathrm{m} \mathrm{s}^{-1} \mathrm{day}^{-1}\right]$ & $0.0017 \pm 0.0018$ & - & \multicolumn{2}{|c|}{$0.0531 \pm 0.0087$} & fitted \\
\hline$\ddot{\gamma}\left[\mathrm{m} \mathrm{s}^{-1} \mathrm{day}^{-2}\right]$ & $-2.03 \pm 0.21 \times 10^{-5}$ & - & - & - & fitted \\
\hline$M \sin i\left[M_{\mathrm{Jup}}\right]$ & $0.597 \pm 0.021^{a}$ & $\geq 1.8$ & $0.259 \pm 0.014$ & $1.370 \pm 0.053$ & derived \\
\hline Radius $R\left[R_{\mathrm{Jup}}\right]$ & $1.019 \pm 0.031$ & - & - & - & derived \\
\hline Density $\rho\left[\mathrm{g} \mathrm{cm}^{-3}\right]$ & $0.70_{-0.064}^{+0.072}$ & - & - & - & derived \\
\hline Surface gravity $\log g$ [cgs] & $3.15 \pm 0.03$ & - & - & - & derived \\
\hline$a[\mathrm{AU}]$ & $0.03673 \pm 0.00064$ & - & $0.1344 \pm 0.0025$ & $0.4756 \pm 0.0087$ & derived \\
\hline
\end{tabular}

Notes. The results for XO-2Nb are discussed in Sect. 5.1, while those for the XO-2S planets are taken from Desidera et al. (2014). We also include our estimates of a few parameters for a second companion to XO-2N, namely XO-2Nc, although its existence is a matter of debate, as discussed in Sect. 5.1.1. ${ }^{(*)}$ See Sect. 5.1.1 for our discussion about the existence of this companion. ${ }^{(a)}$ For XO-2Nb this corresponds to the real mass of the planet. It was obtained with a Monte Carlo analysis using the median values of the orbital period and inclination angle of the orbital plane listed in Table 5, the stellar mass in Table 2 (second value), and the amplitude $\mathrm{K}$ of the radial velocity included in this table.

tilted, but they obtained a poorly determined estimate for the projected spin-orbit angle, $\lambda=10^{\circ} \pm 72^{\circ}$. Here we present updated results obtained from the analysis of 18 spectra collected at that epoch, starting $\sim 1 \mathrm{~h}$ before the beginning of the transit up to $\sim 1 \mathrm{~h}$ after the last contact. The RML effect was modelled with the fitting algorithm described in Covino et al. (2013) and Esposito et al. (2014). Figure 14 shows the RV measurements used for the analysis of the RML effect, folded according to the ephemeris calculated in this work, with the best fit superposed. We found a projected spin-orbit angle $\lambda=7^{\circ} \pm 11^{\circ}$, and derived for the projected rotational velocity of the host star $V \sin I_{\star}=1.07 \pm 0.09 \mathrm{~km} \mathrm{~s}^{-1}$. The $\chi^{2}$ map in the $\lambda-V \sin I_{\star}$ parameter space is shown in the same figure. The uncertainties were derived with a Monte Carlo analysis taking into account the uncertainties of all the parameters used. Our result provides stronger evidence for a spin-orbit alignment with an uncertainty $\sim 7$ times lower. In fact, our new estimate of the transit impact parameter partially breaks the existing degeneracy between $\lambda$ and the stellar projected rotational velocity $V \sin I_{\star}$, resulting in a better constraint on $\lambda$.

\subsection{The $X O-2 N$ spin-orbit alignment in three dimensions}

Thanks to the photometric measurement of the $\mathrm{XO}-2 \mathrm{~N}$ rotation period, together with the analysis of transit photometry and the RML effect, we are able to provide an estimate of the angle $\psi$ between the stellar rotation axis and the normal to the planetary orbital plane. This is given by the formula $\cos \psi=$ $\cos I_{\star} \cos i+\sin I_{\star} \sin i \cos \lambda$ (see discussion in Sect. 4.4 of Winn et al. 2007), where $I_{\star}$ is the inclination between the stellar spin axis and the direction of the line of sight, $i$ is the orbital inclination angle as measured by transit photometry, and $\lambda$ is the projected spin-orbit angle derived from the analysis of the RML effect. By calculating sin $I_{\star}$ from the projected rotational velocity of the star $V \sin I_{\star}$, the rotation period (41.6 \pm 1.1 days), and the more precise measurement of the stellar radius that we derived $\left(0.998 \pm 0.033 R_{\odot}\right)$, we obtain $\sin I_{\star}=0.88 \pm 0.09$. By propagating the errors, we finally obtain $\cos \psi=0.89 \pm 0.12$, as expected very similar to $\sin I_{\star}$ because the angles $i$ and $\lambda$ are close to $90^{\circ}$ and $0^{\circ}$. By neglecting the unphysical values for which $\cos \psi>1$, this result corresponds to $\psi=27_{-27}^{+12}$ degrees. Because a measurement of the angle $\psi$ is difficult to make, out of the 88 planet host stars for which $\lambda$ was determined, $\psi$ was only constrained for nine of them ${ }^{10}$. For $\mathrm{XO}-2 \mathrm{~N}$, our estimate is the first available.

\section{Stellar activity}

In this work we characterize the stellar activity of the XO2 companions, both through photometric measurements and

\footnotetext{
${ }^{10}$ http://WwW . astro.keele.ac .uk/jkt/tepcat/rossiter. html
} 
Table 7. Radial velocities (RV), bisector velocity span (BIS), and activity index $\log \left(R_{\mathrm{HK}}^{\prime}\right)$ of XO-2N, as measured with the HARPS-N spectrograph.

\begin{tabular}{|c|c|c|c|c|c|}
\hline $\begin{array}{c}\text { Time } \\
\text { (BJD UTC }-2450000)\end{array}$ & $\begin{array}{c}\mathrm{RV} \\
\left(\mathrm{km} \mathrm{s}^{-1}\right)\end{array}$ & $\begin{array}{l}\text { RV error } \\
\left(\mathrm{km} \mathrm{s}^{-1}\right)\end{array}$ & $\begin{array}{c}\text { BIS } \\
\left(\mathrm{km} \mathrm{s}^{-1}\right)\end{array}$ & $\log \left(R_{\mathrm{HK}}^{\prime}\right)$ & Error $\log \left(R_{\mathrm{HK}}^{\prime}\right)$ \\
\hline 6252.773594 & 47.0169 & 0.0047 & 0.0209 & -5.172 & 0.157 \\
\hline 6266.500236 & 46.9521 & 0.0018 & 0.0204 & -4.871 & 0.022 \\
\hline 6266.511139 & 46.9500 & 0.0016 & 0.0213 & -4.870 & 0.019 \\
\hline 6266.522077 & 46.9472 & 0.0019 & 0.0187 & -4.905 & 0.026 \\
\hline 6266.533015 & 46.9416 & 0.0018 & 0.0164 & -4.907 & 0.025 \\
\hline 6266.543953 & 46.9406 & 0.0021 & 0.0210 & -4.927 & 0.033 \\
\hline 6266.554891 & 46.9490 & 0.0028 & 0.0303 & -4.874 & 0.043 \\
\hline 6266.565829 & 46.9422 & 0.0024 & 0.0245 & -4.891 & 0.035 \\
\hline 6266.576768 & 46.9413 & 0.0028 & 0.0249 & -4.986 & 0.054 \\
\hline 6266.587706 & 46.9388 & 0.0023 & 0.0150 & -4.889 & 0.034 \\
\hline 6266.598644 & 46.9305 & 0.0023 & 0.0229 & -4.855 & 0.031 \\
\hline 6266.609593 & 46.9217 & 0.0022 & 0.0274 & -4.881 & 0.030 \\
\hline 6266.620520 & 46.9181 & 0.0018 & 0.0195 & -4.889 & 0.023 \\
\hline 6266.631458 & 46.9153 & 0.0017 & 0.0205 & -4.910 & 0.023 \\
\hline 6266.642396 & 46.9162 & 0.0016 & 0.0178 & -4.901 & 0.019 \\
\hline 6266.653334 & 46.9196 & 0.0015 & 0.0209 & -4.872 & 0.017 \\
\hline 6266.664284 & 46.9179 & 0.0016 & 0.0217 & -4.901 & 0.020 \\
\hline 6266.675210 & 46.9135 & 0.0015 & 0.0191 & -4.890 & 0.017 \\
\hline 6266.686148 & 46.9138 & 0.0015 & 0.0152 & -4.877 & 0.017 \\
\hline 6297.623295 & 46.9958 & 0.0015 & 0.0154 & -4.928 & 0.019 \\
\hline 6298.643938 & 46.8409 & 0.0022 & 0.0097 & -4.921 & 0.034 \\
\hline 6299.762797 & 47.0128 & 0.0015 & 0.0099 & -4.907 & 0.020 \\
\hline 6305.631219 & 46.9708 & 0.0020 & 0.0125 & -4.938 & 0.031 \\
\hline 6322.563804 & 46.8777 & 0.0015 & 0.0129 & -4.898 & 0.019 \\
\hline 6323.686453 & 47.0147 & 0.0033 & 0.0209 & -4.947 & 0.072 \\
\hline 6380.349341 & 46.9211 & 0.0033 & 0.0106 & -5.269 & 0.155 \\
\hline 6400.444868 & 46.8469 & 0.0100 & 0.0573 & -4.861 & 0.214 \\
\hline 6406.361875 & 46.8938 & 0.0036 & 0.0113 & -4.908 & 0.060 \\
\hline 6420.392578 & 47.0089 & 0.0031 & 0.0066 & -5.229 & 0.114 \\
\hline 6437.372927 & 46.8412 & 0.0014 & 0.0140 & -4.964 & 0.018 \\
\hline 6548.731988 & 47.0043 & 0.0024 & 0.0059 & -4.949 & 0.044 \\
\hline 6586.684893 & 46.8605 & 0.0024 & 0.0145 & -4.989 & 0.044 \\
\hline 6602.751791 & 46.9259 & 0.0025 & 0.0094 & -5.042 & 0.055 \\
\hline 6616.709751 & 47.0041 & 0.0027 & 0.0203 & -4.874 & 0.042 \\
\hline 6617.722042 & 46.8368 & 0.0017 & 0.0153 & -4.929 & 0.023 \\
\hline 6631.704304 & 46.9647 & 0.0020 & 0.0051 & -5.035 & 0.041 \\
\hline 6657.453439 & 46.8928 & 0.0056 & 0.0255 & -5.086 & 0.183 \\
\hline 6700.404020 & 47.0066 & 0.0026 & 0.0147 & -4.983 & 0.051 \\
\hline 6701.349611 & 46.8410 & 0.0019 & 0.0164 & -4.963 & 0.031 \\
\hline 6783.385415 & 46.9513 & 0.0014 & 0.0077 & -4.996 & 0.024 \\
\hline 6784.360848 & 46.9543 & 0.0016 & 0.0082 & -4.980 & 0.028 \\
\hline 6933.733982 & 46.9031 & 0.0023 & 0.0052 & -5.009 & 0.050 \\
\hline 6935.754527 & 47.0090 & 0.0017 & 0.0107 & -4.996 & 0.030 \\
\hline
\end{tabular}

Notes. The BIS listed here are those derived by the data reduction pipeline of HARPS-N, with uncertainties assumed to be twice those on the radial velocities.

spectroscopic analysis. The analysis of the photometric time series resulted in an estimate of the spin rotation periods for the two components (Sect. 4); we then used the HARPS-N spectra to calculate the chromospheric activity index $R_{\mathrm{HK}}^{\prime}$ based on the analysis of the Ca II H\&K lines. Moreover, an analysis of different CCF line shape parameters (asymmetry indicators) was carried out to look for periodical modulations and correlations with the radial velocities and the $R_{\mathrm{HK}}^{\prime}$ index.

Figueira et al. (2013) introduced and compared several indicators for the asymmetry of the CCF and showed how they can be used to establish whether the radial velocity variation of a star is due to line profile distortions induced by stellar activity, for example. We have computed the bisector inverse span (BIS $)^{11}$, radial velocity difference $\Delta V$, and velocity asymmetry $V_{\text {asy }}$ for each of the XO-2 components, and a comparison between these parameters and the activity index was also performed. We adopted procedures similar to those of Figueira et al. (2013) and Santos et al. (2014) to compute the asymmetry

11 We have verified that between the line-bisector calculated by the DRS and the BIS indicator calculated with the method of Figueira et al. (2013) there is a very high correlation $(R=0.99)$ for both stars; we decided to adopt the latter in our discussion. 


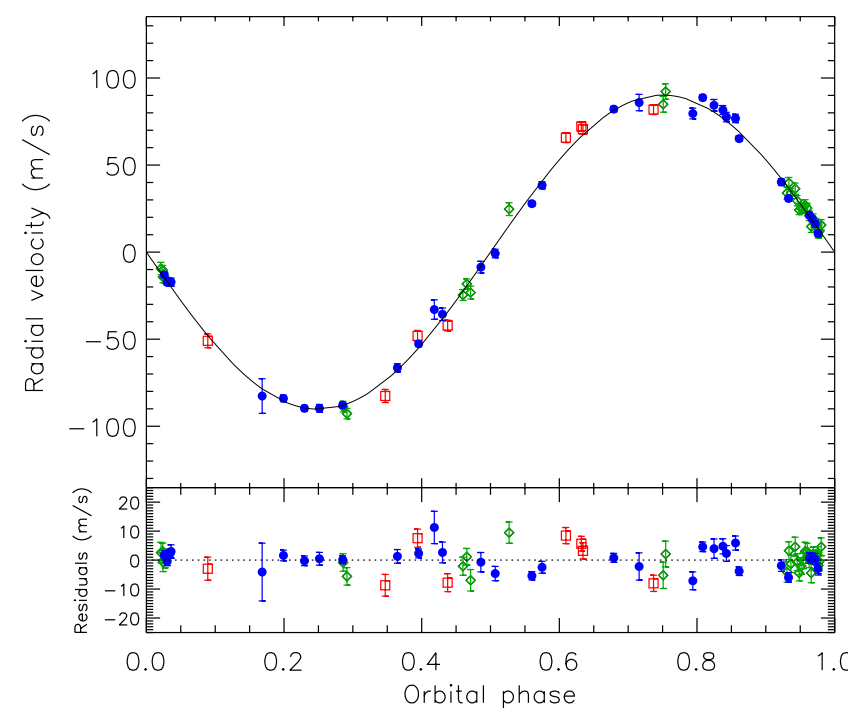

Fig. 10. Phase-folded radial velocity curve of $\mathrm{XO}-2 \mathrm{~N}$ b, after removing the quadratic trend. Green diamonds, red squares, and blue circles indicate the HDS, HIRES, and HARPS-N measurements, respectively. The black solid line shows the best-fit model.

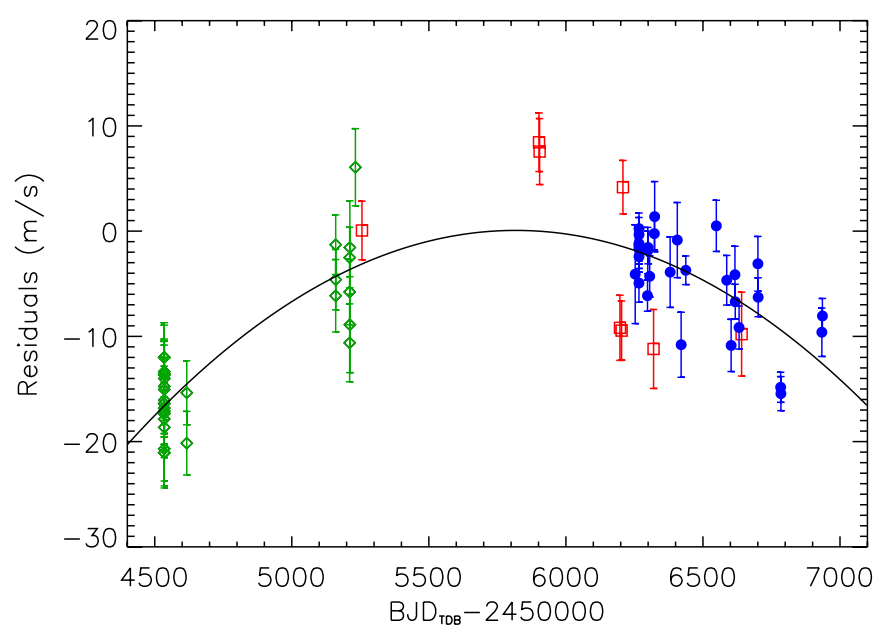

Fig. 11. Long-term trend found in the RV residuals data of $X O-2 \mathrm{~N}$ after removing the orbital signal of $\mathrm{XO}-2 \mathrm{Nb}$, caused either by a longperiod companion $\mathrm{XO}-2 \mathrm{~N}$ c or by the activity cycle of the star. Green diamonds, red squares, and blue circles indicate the HDS, HIRES, and HARPS-N measurements, respectively, while the black solid line shows the best fit of the quadratic trend. Residuals of RV measurements with an error bar greater than $5 \mathrm{~m} \mathrm{~s}^{-1}$, even though considered in the analysis, were not included in this plot for a better visual representation of the long-term trend.

parameters with the exception of $V_{\text {asy }}$ for which the derivative of the CCF with respect to the RV was numerically evaluated by means of a Savitzky-Golay filter (e.g. Press et al. 1992). We estimated the standard deviation of the indicator $\Delta V$ from the standard deviations of the best-fit RVs as obtained with the Levenberg-Marquardt IDL procedure MPFIT . PRO applied to fit Gaussian and bi-Gaussian profiles to the CCF, respectively. The standard deviation of $V_{\text {asy }}$ was computed with a bootstrap procedure by taking into account both the photon shot noise and the correlated noise of the CCF.

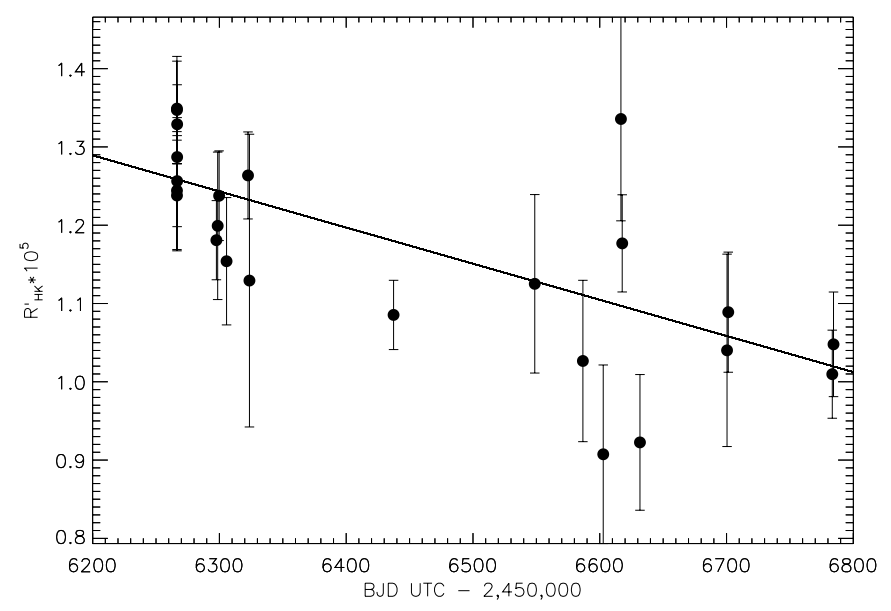

Fig. 12. Time series of the chromospheric activity index $R_{\mathrm{HK}}^{\prime}$ for XO-2N extracted from the HARPS-N spectra. Values of $\log \left(R_{\mathrm{HK}}^{\prime}\right)$ are listed in Table 7. We considered only the spectra with $\mathrm{S} / \mathrm{N}$ greater than 4 in the sixth order. Superposed is the best-fit linear function, revealing the presence of a negative trend.

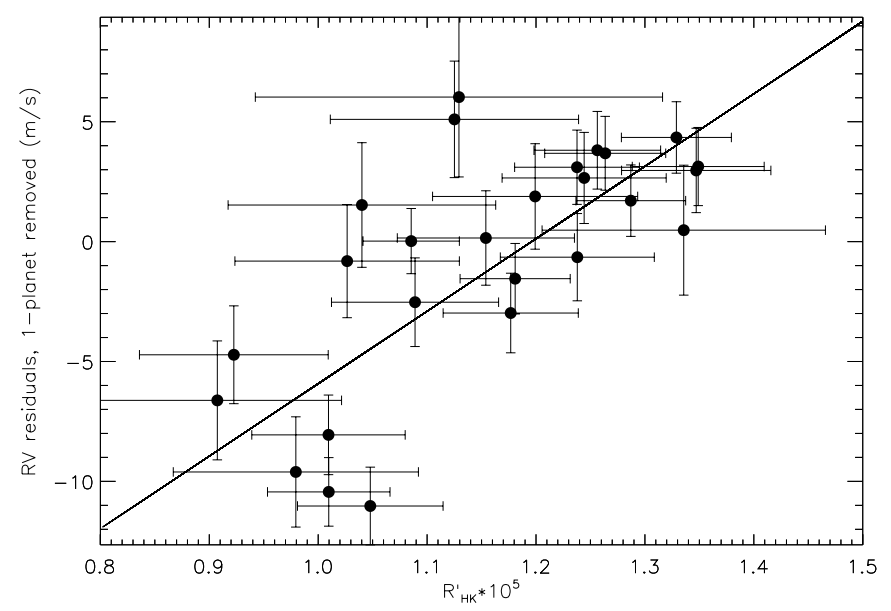

Fig. 13. Correlation between the RV residuals and the chromospheric activity index $R_{\mathrm{HK}}^{\prime}$ for XO-2N. Data are extracted from the HARPS-N spectra. Superposed is the best-fit linear function, showing the existence of a positive trend.

\section{1. $X O-2 N$}

The analysis of the $R_{\mathrm{HK}}^{\prime}$ index time series is presented in Sect. 5.1, where we discuss the correlation found with the RV residuals, possibly indicating that $R_{\mathrm{HK}}^{\prime}$ is outlining the activity cycle of the star. Here we investigate the correlations between the asymmetry indicators and the residuals of the radial velocities (i.e. removing the one-planet fit and the quadratic term), and the results are shown in the left panel of Fig. 15, while the right panel shows the correlations between the same indicators and the activity index $R_{\mathrm{HK}}^{\prime}$. We note that between the CCF asymmetry indicator $\Delta V$ and the $\mathrm{RV}$ residuals there is a moderate and low-FAP correlation, as quantified by the Spearman's rank correlation coefficients indicated at the top of each plot. More significant correlations are observed when considering the $R_{\mathrm{HK}}^{\prime}$ index instead of the RV residuals, in particular for the bisector BIS and $\Delta V$. These seem to indicate that the activity of the star does influence the spectral line profile to some extent.

To better characterize the significance of these correlations, we performed the same analysis as described in Sect. 5.1.1 and based on two different tests. The results are summarized in 

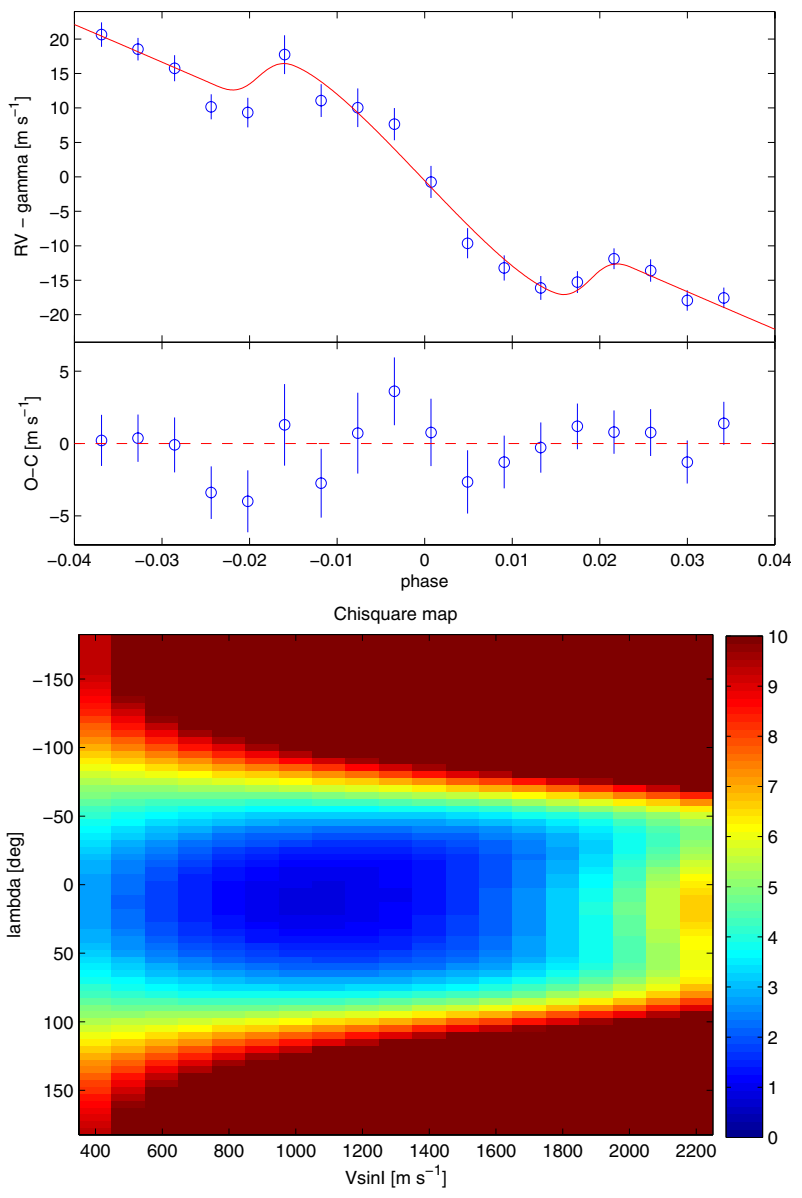

Fig. 14. Upper panel: radial velocity variations of the $\mathrm{XO}-2 \mathrm{~N}$ star in correspondence to the transit of the exoplanet $\mathrm{XO}-2 \mathrm{Nb}$ occurred on the night between 4 and 5 December, 2012. Data are folded according to the transit ephemeris derived in this study $\left[T_{\mathrm{c}}=2455,565.546480+\right.$ $\mathrm{N}^{*} 2.6158592$ BJD TDB], and superposed is our best-fit RML model (red line). Lower panel: the $\chi^{2}$ map in the $\lambda-V \sin I_{\star}$ parameter space.

Table 9. They show that the null hypothesis can be discarded for substantially all the pairs, assuming $\sim 1 \%$ as the threshold for the $p$-value below which a correlation may be assumed significant. The analysis with the composite method suggests that three most significant correlations found by simply computing the Spearman's rank coefficient with the actual datasets, i.e. those between the RV residuals and the BIS/ $\Delta V$ asymmetry indicators of the $\mathrm{CCF}$, are likely less pronounced given the limited number of measurements $(N=25)$. Nonetheless, our analysis suggests that effects of the stellar activity are influencing the spectral line profiles.

We finally analysed the time series of the CCF asymmetry indicators with the GLS algorithm to search for signals related to the stellar rotation period of $\sim 41$ days measured via photometry. No significant peaks were found and, in particular, no periodicity related to the star rotation appears for $R_{\mathrm{HK}}^{\prime}$.

\section{2. $X O-2 S$}

By looking at the average value of the activity index $R_{\mathrm{HK}}^{\prime}$ (Table 8), this star appears less active than its companion. In Fig. 16 the time series of $R_{\mathrm{HK}}^{\prime}$ is shown. The internal errors are comparable to the data scatter, with no clear evidence of a long-term trend.
In the same way as for $\mathrm{XO}-2 \mathrm{~N}$, we investigated the relations between the asymmetry indicators and the $R_{\mathrm{HK}}^{\prime}$ index, and the results are shown in Fig. 17. No significant positive or negative correlation is found, supporting the scenario of a very quiet star. This outcome is confirmed by the results, shown in Table 10, of the analysis performed to asses the significance of the Spearman's rank correlation coefficients, as done for XO-2N. For completeness, we also quantified the correlation between the $R_{\mathrm{HK}}^{\prime}$ index and the RV residuals (after the subtraction of the twoplanet Keplerian solution) calculated by Desidera et al. (2014), obtaining a Spearman's rank coefficient $\rho=0.27$. By testing the null hypothesis, we found a $z$-score $=-0.94$ that corresponds to a significant probability $p=17.4 \%$ that the two datasets are not correlated. This result is confirmed by applying the composite method, from which we obtain $\rho=0.20 \pm 0.15$, indicating lack of correlation.

We also used the GLS algorithm to search for periodicities in the CCF asymmetry indexes and $R_{\mathrm{HK}}^{\prime}$ time series, but no significant peaks were detected. For instance, for the BIS and $\Delta V$ parameters we found peaks close to half the tentative stellar rotation period derived from photometry ( $P \sim 26$ days). A bootstrap analysis resulted in FAP of 0.12 and 0.03 , suggesting that the signals are spurious. No peaks are found close to the orbital periods of the two planets orbiting $\mathrm{XO}-2 \mathrm{~S}$.

\subsection{Comparison of the activity levels for the XO-2 stars}

In Table 8 we summarize our activity-related measurements for the XO-2 components. We can conclude that, at least in the time span covered by our observations, the star XO-2N appears to be slightly more active than the companion. This is also directly visible when comparing the Ca II H\&K lines of the coadded HARPS-N spectra (Fig. 18), which show a greater emission flux in the line cores for the northern component. In addition, the differences seen in the existing correlations between the $\mathrm{CCF}$ asymmetry indicators and the activity index $R_{\mathrm{HK}}^{\prime}$ support the scenario that the activity level of the northern component is higher. This appears to agree with the higher levels of magnetic activity in stars with hot Jupiters with respect to their wide binary companions, as found in particular for HD 189733 and Corot-2 (see e.g. Lanza 2011; Husnoo et al. 2012; Poppenhaeger et al. 2013; Poppenhaeger \& Wolk 2014). Then, the different activity levels of the two stars might be due to an influence of the hot Jupiter on the activity of XO- $2 \mathrm{~N}$ or to the fact that we simply observed the two stars at different epochs of their activity cycles.

In Table 8 we also show the expected values of the spin rotation periods derived from the measurements $\log \left(R_{\mathrm{HK}}^{\prime}\right)$ index according to the works of Noyes et al. (1984) and Mamajek \& Hillenbrand (2008). It can be seen that the estimate of the rotation period of $\mathrm{XO}-2 \mathrm{~N}$ derived from photometry agrees very closely with the expected values.

\section{Long-term stability and evolution of the XO-2 planetary systems}

In a recent work, Wang et al. (2014) determined the planet occurrence rate in multiple-star systems by measuring the stellar multiplicity in a sample of Kepler planet host stars, and comparing the stellar multiplicity rate between these and field stars in the solar neighbourhood. Interestingly, they find that planet formation appears to be significantly suppressed in multiple-star systems when the separation between the planet host star and the other stellar companion is smaller than $1500 \mathrm{AU}$, and this 
Table 8. Summary of stellar activity-related information for the $\mathrm{XO}-2$ system.

\begin{tabular}{ccc}
\hline \hline Parameter & XO-2N & XO-2S \\
\hline $\log \left(R_{\mathrm{HK}}^{\prime}\right)$ & $-4.91 \pm 0.01$ & $-5.02 \pm 0.01$ \\
Exp. stellar rot. period [days] & $39.6 \pm 0.6[\mathrm{~N} 84]$ & $41.1 \pm 0.7[\mathrm{~N} 84]$ \\
& $41.8 \pm 0.8[\mathrm{M} 08]$ & $44.7 \pm 0.7[\mathrm{M} 08]$ \\
Meas. stellar rot. period [days] & $41.6 \pm 1.1$ & $26.0 \pm 0.6$ (or 34.5 days) \\
\hline
\end{tabular}

Notes. The values of the activity index $\log \left(R_{\mathrm{HK}}^{\prime}\right)$ are given as weighted mean, and the uncertainty of each parameter is the standard deviation of the mean. The estimates for the stellar rotation periods correspond to two different relations, those of Noyes et al. (1984) [N84] and Mamajek \& Hillenbrand (2008) [M08].

distance could be thus assumed as the radius of influence of stellar companions. Nonetheless, the estimate of the planet occurrence rate in multiple-star systems is presently a subject of debate. Horch et al. (2014) investigated the binary fraction of Kepler exoplanet host stars by observing more than 600 objects of interest, and conclude that this appears to be similar to the rate observed for field stars (40-50\%), pointing out that the impact of binarity on the planet occurrence should be lower than that emerging from the work of Wang et al. (2014).

The analysis of the stellar properties of the XO-2 binary points to the scenario that the components have a common origin and have been in a bound system since their birth. Although the parameters of the binary orbit are not known, we have performed a basic analysis of the long-term stability of the whole system based on the work of Holman \& Wiegert (1999), extending to the XO-2S system similar considerations done by Burke et al. (2007) for XO-2N. Holman \& Wiegert (1999) modelled the influence of a stellar companion on the stability of a "test particle" orbiting a star with an empirical relation (see Eq. (1) therein) among the stellar mass ratio $\mu=m_{2} /\left(m_{1}+m_{2}\right)$ (the subscript 1 identifies the star orbited by a planet), the binary eccentricity $e$, and the semi-major axis of the binary. This relation provides an estimate of the critical semi-major axis $a_{\mathrm{c}}$, i.e. the maximum orbital distance of a planet to survive after 10000 binary orbits (corresponding to more than $10^{9} \mathrm{yr}$ in the case of $\mathrm{XO}-2$ ).

The work of Holman \& Wiegert (1999) considers planets that orbit only one component of the stellar system, on initially circular and prograde orbits in the plane of the binary. We can apply their relation to each XO-2 star separately, obtaining similar results, such as a mass ratio of $\mu \sim 0.5$ in both cases. By assuming the measured projected binary separation $a=4600 \mathrm{AU}$ as an estimate of the actual semi-major axis of the orbit, and with the eccentricity of the binary orbit left undetermined, we can estimate the critical distance in the range of validity of the empirical formula, $0 \leqslant e \leqslant 0.8$. In the case of a single planet orbiting one of the components the dependence of the critical semi-major axis $a_{\mathrm{c}}$ on the eccentricity is shown in Fig. 19. Following the discussion in Sect. 5 of Holman \& Wiegert (1999), we can assume half of the $a_{\mathrm{c}}$ values found in the case of the three-body problem as a rough estimate of the critical distance when one star hosts more than one planet, which is the case here of the star XO-2S. This result is shown by the thin line in Fig. 19, which shows that if the binary has the highest eccentricity considered here $(e=0.8)$, a planetary companion around $\mathrm{XO}-2 \mathrm{~S}$ would be in a stable orbit if located at a distance $\leqslant 90 \mathrm{AU}$ from the star, while for XO-2N the upper limit is $\sim 170 \mathrm{AU}$.
A final consideration should be made about the possible role of the Lidov-Kozai (L-K) mechanism (e.g. Innanen et al. 1997; Takeda et al. 2008) in particular on the migration of the planet $\mathrm{XO}-2 \mathrm{Nb}$ so close to its parent star. Because the orbital parameters of the binary are not known, at this stage we cannot reconstruct the evolutionary history of this fairly old system. In particular, we do not have any direct measurements of the mutual inclination between them and the binary orbital plane, on which the effects of the L-K mechanism strongly depends. Neveu-VanMalle et al. (2014) suggested that the L-K mechanism likely played a role in shaping the orbits of the two hot Jupiter systems discovered around the components of the WASP-94 binary, this scenario being reinforced by the high projected spin-orbit angle $\lambda=151^{\circ} \pm 20^{\circ}$ and the retrograde orbit of WASP-94Ab. Any discussion about the evolution of the XO-2 planetary systems still remains mostly at the level of speculation. However, by assuming that the orbital configuration of the binary did not change greatly with time, we think the L$\mathrm{K}$ mechanism alone cannot explain why $\mathrm{XO}-2 \mathrm{Nb}$ migrated close to its parent star and became a hot Jupiter, while XO-2Sb and $\mathrm{XO}-2 \mathrm{Sc}$ are farther out, maybe being prevented from migrating because of mutual secular perturbations. In fact, the large separation of the two stars leads to very long periods of the L-K cycle, well beyond the present age of the system. Nonetheless, if the system had originated closer to the galactic bulge, as discussed in Sect. 3.3, this could have produced a strong modification of its orbit with time, making the study of the perturbing mechanisms very complicated. Despite our present inability to assess the role of the L-K mechanism for XO-2 on a firm basis, it is interesting to mention the relevant work of Jensen \& Akeson (2014) about the determination of the protoplanetary disk geometry in a binary system. Thanks to observations with the Atacama Large Millimeter Array (ALMA), they have obtained the clearest picture ever seen of protoplanetary disks in a young binary (age $<4$ Myr), located roughly at the same distance of the XO-2 system. They have provided evidence for a relative inclination of the disks in the range $60^{\circ}-68^{\circ}$, revealing that both or at least one of them must be misaligned with the binary orbital plane, and probably forms an angle far greater than the critical value of $\sim 40^{\circ}$ for which the L-K mechanism is expected to be effective. The results of Jensen \& Akeson (2014) suggest that the formation processes of a binary can favour those necessary conditions to perturb at some extent the planetary orbits since the first stages of planet formation.

\section{Conclusions}

We have investigated in detail the physical properties of the XO-2 stellar and planetary systems. This is the first confirmed case of a wide binary whose components, which have a $T_{\text {eff }}$ similar to our Sun but older and super metal-rich, host planets. This circumstance motivated a first comprehensive characterization study. Our analysis is based on high-resolution spectroscopic data collected with the HARPS-N spectrograph, and new photometric data from several facilities. The main results described in this paper can be summarized as follows:

1. Through a spectroscopic analysis carried out with both the equivalent widths and spectral synthesis methods, and the use of stellar evolutionary tracks, we derived new estimates of the physical parameters of the XO-2 stars. By performing a differential spectral analysis, we measured a positive difference in iron abundance of $\mathrm{XO}-2 \mathrm{~N}$ with respect to $\mathrm{XO}-2 \mathrm{~S}$ at more than $3 \sigma$. When considering this finding in conjunction 

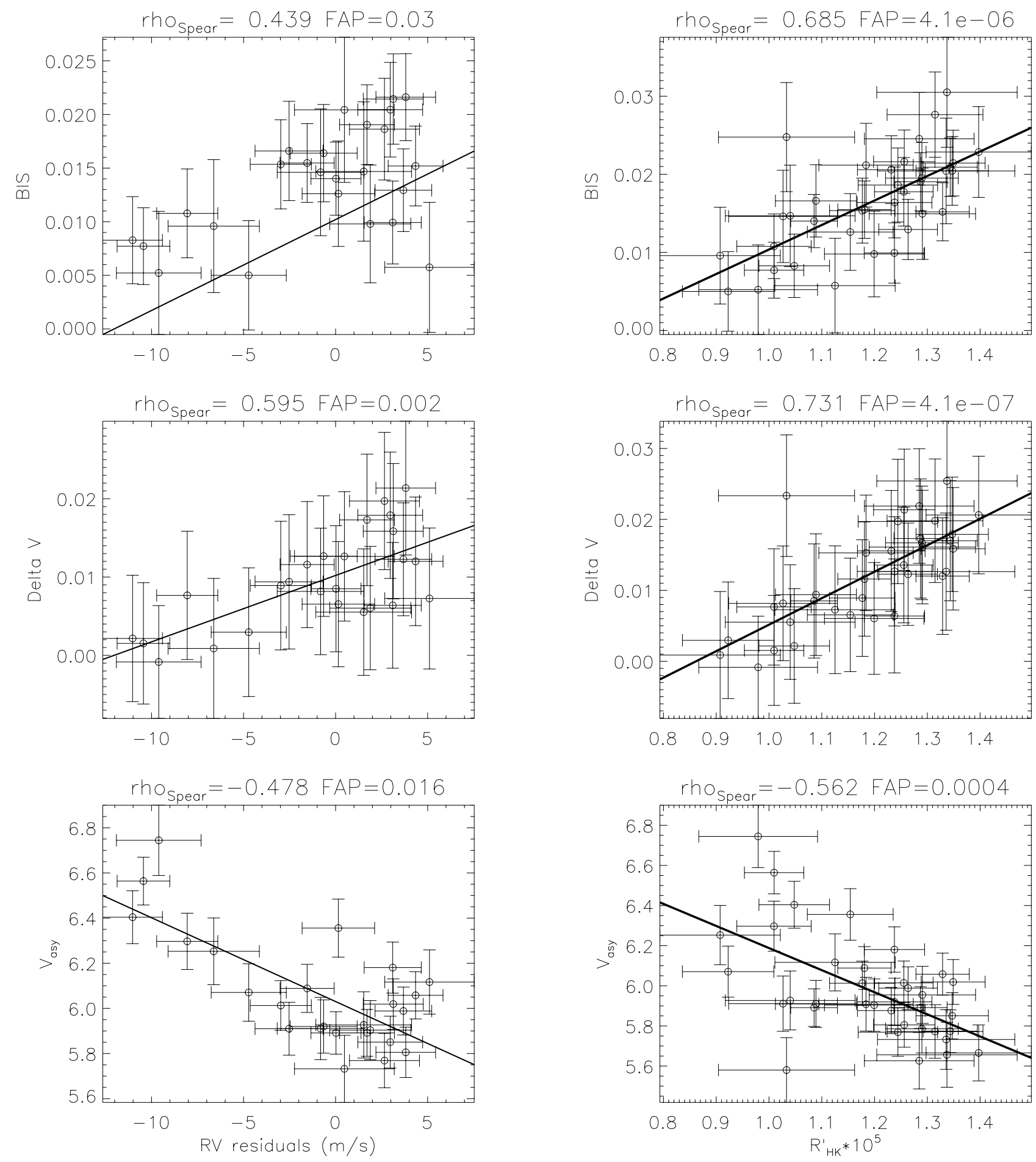

Fig. 15. Correlations between the asymmetry indicators and the residuals of the radial velocities (left panel) for the star XO-2N, and between the same indicators and the activity index $R_{\mathrm{HK}}^{\prime}$ (right panel). We only considered values of $R_{\mathrm{HK}}^{\prime}$ extracted from spectra with $\mathrm{S} / \mathrm{N}$ greater than 4 in the sixth order.

Table 9. Results of the analysis carried out to asses the significance of the Spearman's rank correlation coefficient $\rho$ for the pairs of XO-2N datasets shown in Fig. 15.

\begin{tabular}{|c|c|c|c|c|c|c|}
\hline Method & RV resid. vs. BIS & $\mathrm{RV}$ resid. vs. $\Delta V$ & $\mathrm{RV}$ resid. vs. $V_{\text {asy }}$ & $R_{\mathrm{HK}}^{\prime}$ vs. BIS & $R_{\mathrm{HK}}^{\prime}$ vs. $\Delta V$ & $R_{\mathrm{HK}}^{\prime}$ vs. $V_{\text {asy }}$ \\
\hline \multirow[t]{2}{*}{ Null hypothesis } & $z$-score $=-2.14$ & $z$-score $=-2.93$ & $z$-score $=+2.36$ & $z$-score $=-4.04$ & $z$-score $=-4.32$ & $z$-score $=+3.31$ \\
\hline & $p$-value $=1.6 \%$ & $p$-value $=0.9 \%$ & $p$-value $=0.17 \%$ & $p$-value $=0.003 \%$ & $p$-value $=0.05 \%$ & $p$-value $<0.002 \%$ \\
\hline Composite Monte Carlo & $\rho=+0.32 \pm 0.19$ & $\rho=+0.34 \pm 0.18$ & $\rho=-0.43 \pm 0.19$ & $\rho=+0.44 \pm 0.14$ & $\rho=+0.39 \pm 0.15$ & $\rho=-0.40 \pm 0.15$ \\
\hline Original $\rho$ & +0.685 & +0.731 & -0.562 & +0.439 & +0.595 & -0.478 \\
\hline
\end{tabular}




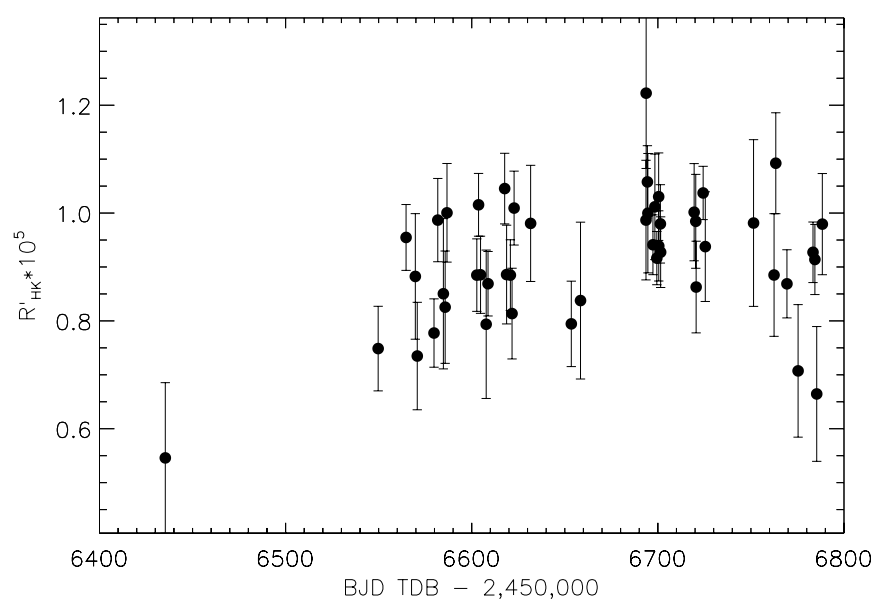

Fig. 16. Time series of the chromospheric activity index $R_{\mathrm{HK}}^{\prime}$ for the star XO-2S. Only values of $R_{\mathrm{HK}}^{\prime}$ derived from spectra with $S / N>3.9$ in the sixth order were considered.

Table 10. Results of the analysis carried out to asses the significance of the Spearman's rank correlation coefficient $\rho$ for the pairs of XO-2S datasets shown in Fig. 17.

\begin{tabular}{cccc}
\hline \hline Method & $R_{\mathrm{HK}}^{\prime}$ vs. BIS & $R_{\mathrm{HK}}^{\prime}$ vs. $\Delta V$ & $R_{\mathrm{HK}}^{\prime}$ vs. $V_{\text {asy }}$ \\
\hline Null hypoth. & $z$-score $=0.45$ & $z$-score $=0.37$ & $z$-score $=0.69$ \\
& $p$-value $=32.6 \%$ & $p$-value $=47.2 \%$ & $p$-value $=24.5 \%$
\end{tabular}

Composite MC $\rho=-0.046 \pm 0.150 \rho=-0.008 \pm 0.145 \rho=-0.047 \pm 0.145$

\begin{tabular}{llll} 
Original $\rho$ & -0.068 & -0.054 & -0.101 \\
\hline
\end{tabular}

with the existence of a hot Jupiter around the star with the higher iron abundance, a very interesting implication is that the evolution of this planetary system, assuming the migration of the giant planet, could have favoured the ingestion by the host star of rocky material in the planetary disk, which can be revealed as an excess of iron abundance.

2. Fourteen new transit light curves were collected of the hot Jupiter XO-2Nb. From their combined analysis we derived updated and very accurate transit parameters. By analysing the out-of-transit light curve of $\mathrm{XO}-2 \mathrm{~N}$ collected during a time span of $\sim 4$ months, we measured its rotation period for the first time. We found that the light curve can be folded almost perfectly with a sine function of period $41.4 \pm 1.1$ days and that our estimate closely agrees with predictions. We also provide an estimate for the rotation period of XO-2S, but our result is inconclusive because the interpretation of the periodogram is more complicated than in the case of $\mathrm{XO}-2 \mathrm{~N}$. The highest peak corresponds to a rotation period of 26 days, which is $\sim 15$ days shorter than that of XO-2N and is far from being in agreement with gyrochronology predictions. However, another peak with almost comparable power is found at 34.5 days and for the moment the true periodicity cannot be determined because of aliasing effects.

3. We analysed the radial velocity time series of the XO- $2 \mathrm{~N}$ star, improving the estimates of the orbital parameters of $\mathrm{XO}-2 \mathrm{Nb}$. We note that we confirmed the existence of an acceleration previously reported in the literature and revealed a turnover in the trend. We discussed the implications of this finding in terms of the presence of an outer companion for which we determined a lower limit for its mass and
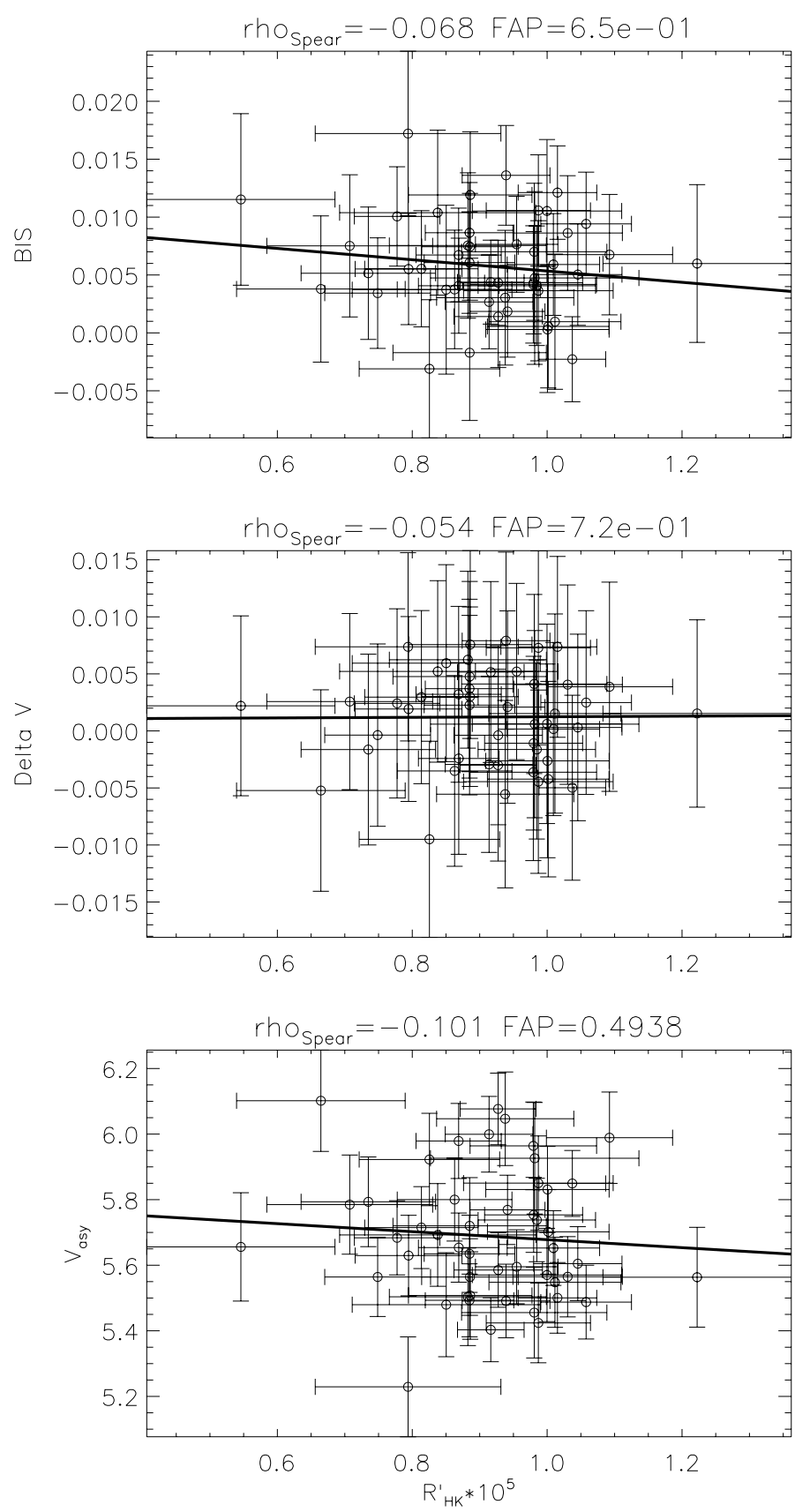

Fig. 17. Correlations between the asymmetry indicators and the chromospheric activity index $R_{\mathrm{HK}}^{\prime}$ for the star XO-2S. Only values of $R_{\mathrm{HK}}^{\prime}$ derived from spectra with $S / N>3.9$ in the sixth order were considered for the analysis.

orbital period. Nonetheless, we found a significant positive correlation between the residuals of the RV and the activity index $R_{\mathrm{HK}}^{\prime}$ which would imply that the observed acceleration is instead caused by the intrinsic activity of the star and related to its solar-like activity cycle. However, although we found relevant and convincing indications for a stellar activity cycle as the real cause of the observed long-term RV variation, more observations would be advisable to definitively clarify the origin of the RV trend. Finally, by combining the results from the analysis of the transit light curves and of the radial velocity time series (archival data and new HARPS-N measurements), we refined the main physical parameters 

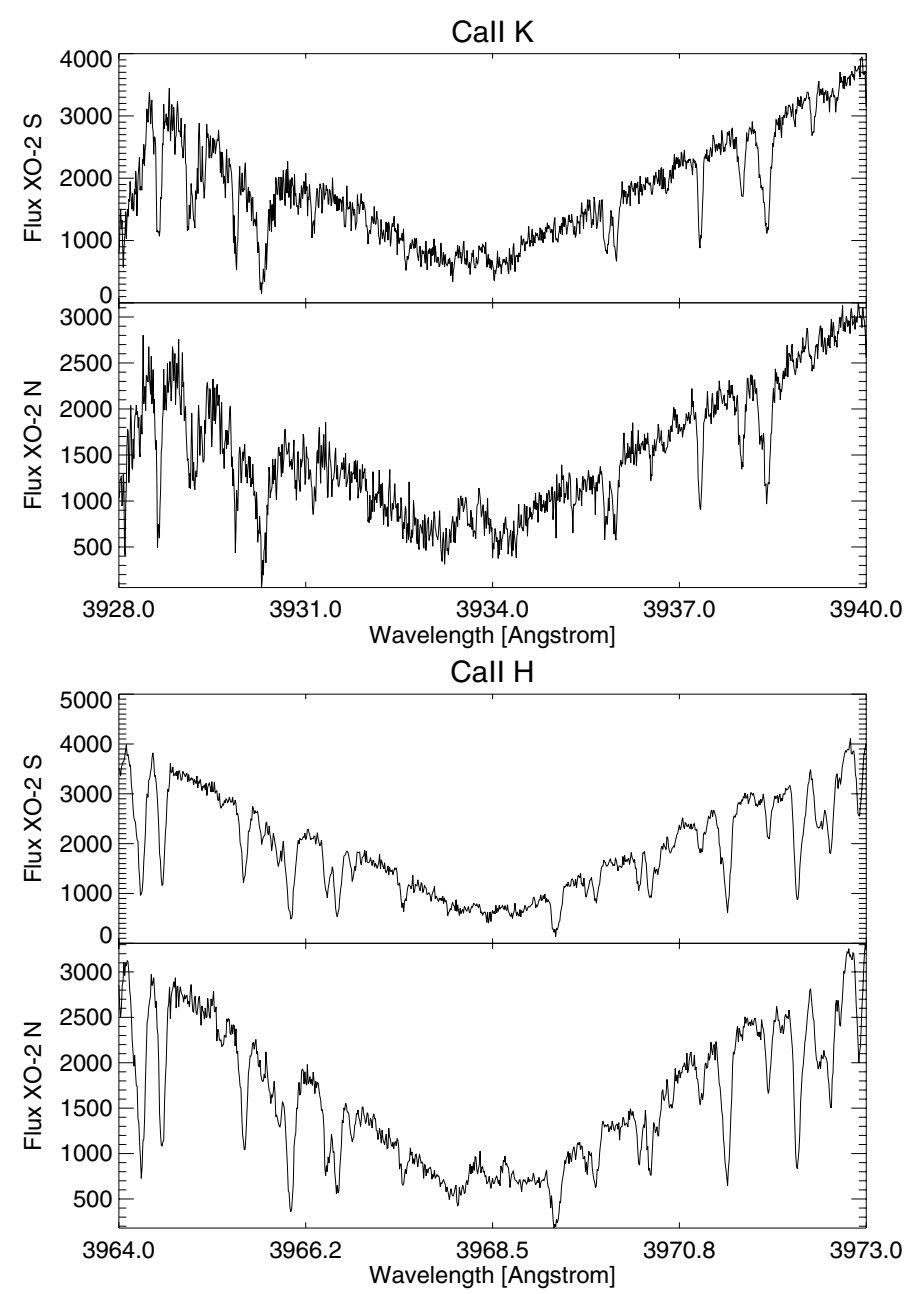

Fig. 18. Ca II K (upper panel) and $\mathrm{H}$ (lower panel) lines of the coadded HARPS-N spectra of XO-2N and XO-2S. A greater emission flux is clearly visible in the line cores of the northern component, which is indicative of this star's higher activity level.

of the planet $\mathrm{XO}-2 \mathrm{Nb}$, in particular the mass, radius, and density.

4. We modelled the Rossiter-McLaughlin effect for $\mathrm{XO}-2 \mathrm{~N}$, providing a better constraint on the projected spin-orbit angle $\lambda=7^{\circ} \pm 11^{\circ}$, according to which the system is likely aligned. By combining the result on $\lambda$ with that on the stellar rotation period, we were able to derive for the first time an upper limit of $39^{\circ}$ for the true angle $\psi$ formed by the stellar rotation axis and the normal to the planetary orbital plane.

5. We investigated the stellar activity of the XO-2 components by analysing a set of asymmetry indicators of the spectral cross-correlation function and the chromospheric activity index $R_{\mathrm{HK}}^{\prime}$. We conclude that the northern component was more active during the time span of our observations, and that the different activity levels might be due to an influence of the hot Jupiter XO- $2 \mathrm{Nb}$ on its host star or to different phases of their activity cycles.

The XO-2 system should be still regarded with interest, in particular for the evidence of long-term trends in the RVs of both the stars for which a clear explanation is still not possible. Should these signals be caused by additional companions in outer orbits, this would make the system even more interesting to be studied as a peculiar laboratory for testing the theories of planet

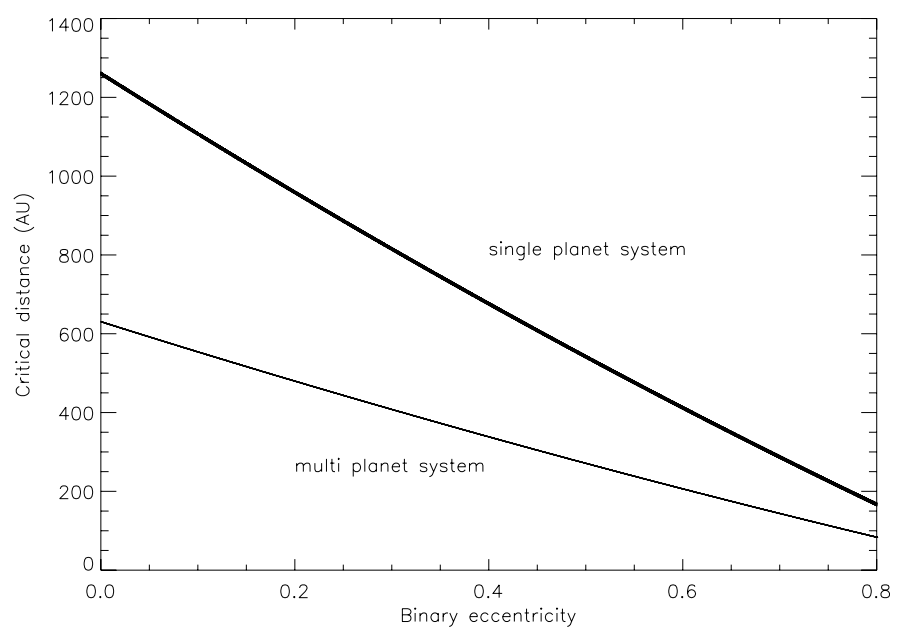

Fig. 19. Critical semi-major axis $a_{\mathrm{c}}$ for each star in the XO-2 binary system, defined as the maximum distance that a single planet, on initially circular orbits, could have from its parent star to survive after $10000 \mathrm{bi}$ nary orbits (thick line). The thin line represents a simple estimate of $a_{\mathrm{c}}$ when one of the stars hosts more than one planet. These results are based on the work of Holman \& Wiegert (1999).

formation and evolution. We are collecting new data to improve the characterization of the whole system and provide insights to several open questions.

Acknowledgements. We thank the anonymous referee for relevant comments which improved the quality of the data analysis. The GAPS project acknowledges support from INAF through the "Progetti Premiali" funding scheme of the Italian Ministry of Education, University, and Research. The Astronomical Observatory of the Autonomous Region of the Aosta Valley is supported by the Regional Government of the Aosta Valley, the Town Municipality of Nus and the Mont Emilius Community. M.D. acknowledges partial support from INAFOATo through the grant "Progetto GAPS: caratterizzazione spettroscopica e fotometrica dei target (attività cromosferica, rotazione) e studio delle sinergie tra GAPS e APACHE" (\#35/2014), and from ASI under contract to INAF I/058/10/0 (Gaia Mission - The Italian Participation to DPAC). J.M.C. and A.B. are supported by a grant of the European Union-European Social Fund, the Autonomous Region of the Aosta Valley and the Italian Ministry of Labour and Social Policy. We thank ASI (through contracts I/037/08/0 and I/058/10/0) and Fondazione CRT for their support to the APACHE Project. V.N. acknowledges partial support from INAF-OAPd through the grant "Analysis of HARPS-N data in the framework of GAPS project" (\#19/2013) and "Studio preparatorio per le osservazioni della missione ESA/CHEOPS" (\#42/2013). NCS acknowledges support from Fundação para a Ciência e a Tecnologia (FCT, Portugal) through FEDER funds in program COMPETE, as well as through national funds, in the form of grants reference RECI/FIS-AST/0176/2012 (FCOMP-01-0124-FEDER-027493), and RECI/FIS-AST/0163/2012 (FCOMP-01-0124-FEDER-027492), and in the form of the Investigador FCT contract reference IF/00169/2012 and POPH/FSE (EC) by FEDER funding through the program "Programa Operacional de Factores de Competitividade - COMPETE". N.C.S. further acknowledges the support from the European Research Council/European Community under the FP7 through Starting Grant agreement number 239953. I.R. acknowledges support from the Spanish Ministry of Economy and Competitiveness (MINECO) and the "Fondo Europeo de Desarrollo Regional" (FEDER) through grants AYA2012-39612C03-01 and ESP2013-48391-C4-1-R. We thank M. Fiaschi and A. Zurlo for their support during the TASTE observations of 2011/02/06 and 2011/04/02, respectively. This research has made use of the SIMBAD database and VizieR catalogue access tool, operated at CDS, Strasbourg, France.

\section{References}

Baranne, A., Queloz, D., Mayor, M., et al. 1996, A\&AS, 119, 373 Barbieri, M., \& Gratton, R. G. 2002, A\&A, 384, 879

Barnes, S. A. 2007, ApJ, 669, 1167

Bertin, E., \& Arnouts, S. 1996, A\&AS, 117, 393

Bianchi, L., Herald, J., Efremova, B., et al. 2011, Ap\&SS, 335, 161

Biazzo, K., Randich, S., \& Palla, F. 2011, A\&A, 525, A35 
Biazzo, K., D’Orazi, V., Desidera, S., et al. 2012, MNRAS, 427, 2905 Bonfils, X., Delfosse, X., Udry, S., et al. 2013, A\&A, 549, A109 Burke, C. J., McCullough, P. R., Valenti, J. A., et al. 2007, ApJ, 671, 2115 Casagrande, L., \& VandenBerg, D. A. 2014, MNRAS, 444, 392 Claret, A., \& Bloemen, S. 2011, A\&A, 529, A75 Coskunoğlu, B., Ak, S., Bilir, S., et al. 2011, MNRAS, 412, 1237 Cosentino, R., Lovis, C., Pepe, F., et al. 2012, in SPIE Conf. Ser., 8446 Covino, E., Esposito, M., Barbieri, M., et al. 2013, A\&A, 554, A28 Crouzet, N., McCullough, P. R., Burke, C., \& Long, D. 2012, ApJ, 761, 7 Curran, P. A. 2014 [arXiv: 1411.3816]

Daemgen, S., Hormuth, F., Brandner, W., et al. 2009, A\&A, 498, 567

Davies, G. R., Chaplin, W. J., Farr, W. M., et al. 2015, MNRAS, 446, 2959

Demarque, P., Woo, J.-H., Kim, Y.-C., \& Yi, S. K. 2004, ApJS, 155, 667

Desidera, S., Gratton, R. G., Scuderi, S., et al. 2004, A\&A, 420, 683

Desidera, S., Gratton, R. G., Lucatello, S., \& Claudi, R. U. 2006, A\&A, 454, 581

Desidera, S., Gratton, R., Endl, M., et al. 2007, Chap. 5 in Planets in

Binary Star Systems, ed. N. Haghighpour (Springer publishing company) [arXiv: 0705.3141]

Desidera, S., Sozzetti, A., Bonomo, A. S., et al. 2013, A\&A, 554, A29

Desidera, S., Bonomo, A. S., Claudi, R. U., et al. 2014, A\&A, 567, L6

Drimmel, R., \& Spergel, D. N. 2001, ApJ, 556, 181

Duchêne, G., \& Kraus, A. 2013, ARA\&A, 51, 269

Dumusque, X., Pepe, F., Lovis, C., et al. 2012, Nature, 491, 207

Eggenberger, A., \& Udry, S. 2010, in Astrophys. Space Sci. Lib., 366, ed.

N. Haghighipour, 19, 366

Esposito, M., Covino, E., Mancini, L., et al. 2014, A\&A, 564, L13

Fabrycky, D., \& Tremaine, S. 2007, ApJ, 669, 1298

Facchini, S., Lodato, G., \& Price, D. J. 2013, MNRAS, 433, 2142

Fernandez, J. M., Holman, M. J., Winn, J. N., et al. 2009, AJ, 137, 4911

Figueira, P., Santos, N. C., Pepe, F., Lovis, C., \& Nardetto, N. 2013, A\&A, 557, A93

Figueira, P., Oshagh, M., Adibekyan, V. Z., \& Santos, N. C. 2014, A\&A, 572, A51

Fragner, M. M., Nelson, R. P., \& Kley, W. 2011, A\&A, 528, A40

Gandolfi, D., Parviainen, H., Fridlund, M., et al. 2013, A\&A, 557, A74

Giacobbe, P., Damasso, M., Sozzetti, A., et al. 2012, MNRAS, 424, 3101

Gratton, R. G., Bonanno, G., Claudi, R. U., et al. 2001, A\&A, 377, 123

Gregory, P. C. 2005, ApJ, 631, 1198

Henden, A., \& Munari, U. 2014, Contributions of the Astronomical Observatory Skalnate Pleso, 43, 518

Holman, M. J., \& Wiegert, P. A. 1999, AJ, 117, 621

Horch, E. P., Howell, S. B., Everett, M. E., \& Ciardi, D. R. 2014, ApJ, 795, 60

Husnoo, N., Pont, F., Mazeh, T., et al. 2012, MNRAS, 422, 3151

Ida, S., \& Lin, D. N. C. 2008, ApJ, 673, 487

Innanen, K. A., Zheng, J. Q., Mikkola, S., \& Valtonen, M. J. 1997, AJ, 113, 1915

Jensen, E. L. N., \& Akeson, R. 2014, Nature, 51, 567

Johnson, J. A., Aller, K. M., Howard, A. W., \& Crepp, J. R. 2010, PASP, 122, 905

Jones, H. R. A., Butler, R. P., Tinney, C. G., et al. 2006, MNRAS, 369, 249

Jordán, A., \& Bakos, G. Á. 2008, ApJ, 685, 543

Kaib, N. A., \& Raymond, S. N. 2014, ApJ, 782, 60

Kaib, N. A., Raymond, S. N., \& Duncan, M. 2013, Nature, 493, 381

Kipping, D. M., Hartman, J., Bakos, G. Á., et al. 2011, AJ, 142, 95

Kley, W., \& Nelson, R. P. 2008, A\&A, 486, 617

Knutson, H. A., Fulton, B. J., Montet, B. T., et al. 2014, ApJ, 785, 126

Kundurthy, P., Barnes, R., Becker, A. C., et al. 2013, ApJ, 770, 36

Lanza, A. F. 2011, Ap\&SS, 336, 303

Lanza, A. F., Rodonò, M., Pagano, I., Barge, P., \& Llebaria, A. 2003, A\&A, 403, 1135

Laws, C., \& Gonzalez, G. 2001, ApJ, 553, 405

Lillo-Box, J., Barrado, D., Henning, T., et al. 2014a, A\&A, 568, L1

Lillo-Box, J., Barrado, D., Moya, A., et al. 2014b, A\&A, 562, A109

Liu, F., Asplund, M., Ramírez, I., Yong, D., \& Meléndez, J. 2014, MNRAS, 442 L51

Lovis, C., Dumusque, X., Santos, N. C., et al. 2011, unpublished [arXiv: 1107.5325]

Machalek, P., McCullough, P. R., Burrows, A., et al. 2009, ApJ, 701, 514

Mack, III, C. E., Schuler, S. C., Stassun, K. G., \& Norris, J. 2014, ApJ, 787, 98

Mamajek, E. E., \& Hillenbrand, L. A. 2008, ApJ, 687, 1264

Marzari, F., \& Scholl, H. 2000, ApJ, 543, 328

Marzari, F., Thébault, P., \& Scholl, H. 2009, A\&A, 507, 505

Marzari, F., Baruteau, C., Scholl, H., \& Thebault, P. 2012, A\&A, 539, A98

Mayor, M., Marmier, M., Lovis, C., et al. 2011, A\&A, submitted [arXiv: 1109.2497]

Meibom, S., Torres, G., Fressin, F., et al. 2013, Nature, 499, 55

Moeckel, N., \& Veras, D. 2012, MNRAS, 422, 831

Mortier, A., Santos, N. C., Sozzetti, A., et al. 2012, A\&A, 543, A45

Mugrauer, M., Ginski, C., \& Seeliger, M. 2014, MNRAS, 439, 1063
Munari, U., Henden, A., Frigo, A., et al. 2014, AJ, 148, 81

Narita, N., Hirano, T., Sato, B., et al. 2011, PASJ, 63, L67

Nascimbeni, V., Piotto, G., Bedin, L. R., \& Damasso, M. 2011, A\&A, 527, A85

Nascimbeni, V., Cunial, A., Murabito, S., et al. 2013, A\&A, 549, A30

Nelson, A. F. 2000, ApJ, 537, L65

Neveu-VanMalle, M., Queloz, D., Anderson, D. R., et al. 2014, A\&A, 572, A49

Noyes, R. W., Hartmann, L. W., Baliunas, S. L., Duncan, D. K., \& Vaughan,

A. H. 1984, ApJ, 279, 763

Ossendrijver, A. J. H. 1997, A\&A, 323, 151

Østensen, R., \& Solheim, J.-E. 2000, Baltic Astron., 9, 411

Pepe, F., Mayor, M., Galland, F., et al. 2002, A\&A, 388, 632

Perryman, M. A. C., \& ESA 1997, in The HIPPARCOS and TYCHO catalogues. Astrometric and photometric star catalogues derived from the ESA HIPPARCos Space Astrometry Mission, ESA SP, 1200

Picogna, G., \& Marzari, F. 2013, A\&A, 556, A148

Pinsonneault, M. H., DePoy, D. L., \& Coffee, M. 2001, ApJ, 556, L59

Poppenhaeger, K., \& Wolk, S. J. 2014, A\&A, 565, L1

Poppenhaeger, K., Schmitt, J. H. M. M., \& Wolk, S. J. 2013, ApJ, 773, 62

Press, W. H., Teukolsky, S. A., Vetterling, W. T., \& Flannery, B. P. 1992, Numerical recipes in FORTRAN. The art of scientific computing (Cambridge University Press)

Quinn, S. N., White, R. J., Latham, D. W., et al. 2012, ApJ, 756, L33

Raghavan, D., McAlister, H. A., Henry, T. J., et al. 2010, ApJS, 190, 1

Ramírez, I., Meléndez, J., Cornejo, D., Roederer, I. U., \& Fish, J. R. 2011, ApJ, 740, 76

Raymond, S. N., Armitage, P. J., Moro-Martín, A., et al. 2011, A\&A, 530, A62

Roell, T., Neuhäuser, R., Seifahrt, A., \& Mugrauer, M. 2012, A\&A, 542, A92

Rowe, J. F., Bryson, S. T., Marcy, G. W., et al. 2014, ApJ, 784, 45

Santerne, A., Díaz, R. F., Bouchy, F., et al. 2011, A\&A, 528, A63

Santos, N. C., Mayor, M., Bonfils, X., et al. 2011, A\&A, 526, A112

Santos, N. C., Sousa, S. G., Mortier, A., et al. 2013, A\&A, 556, A150

Santos, N. C., Mortier, A., Faria, J. P., et al. 2014, A\&A, 566, A35

Schlafly, E. F., \& Finkbeiner, D. P. 2011, ApJ, 737, 103

Schuler, S. C., Cunha, K., Smith, V. V., et al. 2011a, ApJ, 737, L32

Schuler, S. C., Flateau, D., Cunha, K., et al. 2011b, ApJ, 732, 55

Sing, D. K., Désert, J.-M., Fortney, J. J., et al. 2011, A\&A, 527, A73

Sing, D. K., Huitson, C. M., Lopez-Morales, M., et al. 2012, MNRAS, 426, 1663

Skrutskie, M. F., Cutri, R. M., Stiening, R., et al. 2006, AJ, 131, 1163

Sneden, C. 1973, ApJ, 184, 839

Southworth, J. 2008, MNRAS, 386, 1644

Southworth, J. 2011, MNRAS, 417, 2166

Southworth, J., Maxted, P. F. L., \& Smalley, B. 2004, MNRAS, 351, 1277

Sozzetti, A., Torres, G., Charbonneau, D., et al. 2007, ApJ, 664, 1190

Sozzetti, A., Torres, G., Latham, D. W., et al. 2009, ApJ, 697, 544

Sozzetti, A., Bernagozzi, A., Bertolini, E., et al. 2013, in EPJ Web Conf., 47, 3006

Takeda, G., Kita, R., \& Rasio, F. A. 2008, ApJ, 683, 1063

Teske, J. K., Schuler, S. C., Cunha, K., Smith, V. V., \& Griffith, C. A. 2013, ApJ, 768, L12

Thebault, P., \& Haghighipour, N. 2014, in Planetary Exploration and Science: Recent Advances and Applications, eds. S. Jin, N. Haghighipour, \& W.-H. Ip (Springer), in press [arXiv: 1406.1357]

Wang, J., Fischer, D. A., Xie, J.-W., \& Ciardi, D. R. 2014, ApJ, 791, 111

Winn, J. N., Holman, M. J., Henry, G. W., et al. 2007, ApJ, 133, 1828

Zacharias, N., Finch, C. T., Girard, T. M., et al. 2013, AJ, 145, 44

Zechmeister, M., \& Kürster, M. 2009, A\&A, 496, 577

1 INAF-Osservatorio Astrofisico di Torino, via Osservatorio 20, 10025 Pino Torinese, Italy

e-mail: damasso@oato.inaf.it

2 Osservatorio Astronomico della Regione Autonoma Valle d'Aosta, Fraz. Lignan 39, 11020, Nus (Aosta), Italy

3 INAF-Osservatorio Astrofisico di Catania, via S.Sofia 78, 95123 Catania, Italy

4 INAF-Osservatorio Astronomico di Padova, Vicolo dell'Osservatorio 5, 35122 Padova, Italy

5 Dip. di Fisica e Astronomia Galileo Galilei - Università di Padova, Vicolo dell'Osservatorio 2, 35122 Padova, Italy

6 Instituto de Astrofísica de Canarias, C/via Láctea S/N, 38200 La Laguna, Tenerife, Spain

7 Departamento de Astrofísica, Universidad de La Laguna, 38205 La Laguna, Tenerife, Spain

8 Fundación Galileo Galilei - INAF, Rambla José Ana Fernandez Pérez 7, 38712 Breña Baja, TF - Spain 
9 Obs. Astronomique de l'Univ. de Geneve, 51 ch. des Maillettes Sauverny, 1290 Versoix, Switzerland

10 INAF-Osservatorio Astronomico di Brera, via E. Bianchi 46, 23807 Merate (LC), Italy

11 Landessternwarte Königstuhl, Zentrum für Astronomie der Universitat Heidelberg, Königstuhl 12, 69117 Heidelberg, Germany

12 Inst. de Ciencies de l'Espai (CSIC-IEEC), Campus UAB, Facultat de Ciencies, 08193 Bellaterra, Spain

13 Instituto de Astrofísica e Ciências do Espaço, Universidade do Porto, CAUP, Rua das Estrelas, 4150-762 Porto, Portugal

14 Centro de Astrofísica, Universidade do Porto, Rua das Estrelas, 4150-762 Porto, Portugal

15 Departamento de Física e Astronomia, Faculdade de Ciências, Univ. do Porto, Rua do Campo Alegre, s/n, 4169-007 Porto, Portugal
16 INAF-Osservatorio Astronomico di Palermo, Piazza del Parlamento, 1, 90134 Palermo, Italy

17 Dept. of Physics, University of Perugia, via A. Pascoli, 06123 Perugia, Italy

18 INAF-Osservatorio Astronomico di Capodimonte, Salita Moiariello 16, 80131 Napoli, Italy

19 Dipartimento di Fisica, Università di Milano, via Celoria 16, 20133 Milano, Italy

20 Max-Planck-Institut für Astronomie, Königstuhl 17, 69117 Heidelberg, Germany

21 INAF-IASF Milano, via Bassini 15, 20133 Milano, Italy

22 INAF-Osservatorio Astronomico di Trieste, via Tiepolo 11, 34143 Trieste, Italy

23 Astrophysics Group, Keele University, Staffordshire, ST5 5BG, UK 